Without corrections/

ohne Korrekturen

After corrections/

nach Ausführung

der Korrekturen

Date/Datum:

Signature/Zeichen

\title{
5
}

\section{Modeling the Properties of Carbon Nanotubes for Sensor-Based Devices}

Cosmin Roman, Mikro- und Nanosysteme, ETH Zürich, Switzerland Stephan Roche, Commissariat à l'Energie Atomique, Grenoble, France Angel Rubio, Universidad del País Vasco, Centro Mixto, San Sebastián/Donostia, Spain

5.1 Introduction 182

5.2 Properties of Carbon Nanotubes: Reminder 183

5.3 Carbon Nanotube-Based Sensor Demonstrators: State-of-the-Art 186

5.3.1 Modulation of Electronic Properties 186

5.3.2 Shifting of Mechanical Resonances 187

5.3.3 Other Transduction Mechanisms 188

5.4 Modeling: Reviewing Methods and Tools 189

5.4.1 Electronic Structure Calculations 189

5.4.2 Transport Formalisms 191

5.4.3 Mechanical Models 193

5.5 Modeling Case Studies: Highlighting Physical Mechanisms 195

5.5.1 Scanning Tunneling Microscopy of Nanotubes 195

5.5.2 Transport Phenomena in Carbon Nanotubes 199

5.5.2.1 Model Disorder: Basics of Elastic Mean-Free-Path Scaling 199

5.5.2.2 Chemical Disorder or Doping: Conduction Mechanisms and Basic Length Scales 201

5.5.2.3 Defects, Covalent Functionalization and Anderson Localization 204

5.5.2.4 Signatures of Physisorbed Molecules on Transport 207

5.5.2.5 Sensor Specificity 212

5.5.3 An Electromechanical Displacement Transducer 215

5.5.3.1 Mechanical Transducer Behavior 217

5.5.3.2 Transport Strain-Current Characteristic 220

5.6 Conclusions and Perspectives 222

5.7 Acknowledgments 222

5.8 List of Symbols 223

References 223 
5.1 Introduction

At only 16 years since the discovery of carbon nanotubes (CNTs) by Sumio Iijima [1], we are witnessing an explosive development of nanotube science and technology. Considering the rapid progress made in the fabrication, manipulation, characterization and modeling of nanostructures based on nanotubes, it is reasonable to expect that CNTs will, in the next few years, spread to key application areas such as energy, materials and devices. Several structural varieties of nanotubes have been identified and classified based on criteria such as helicity (also known as chirality), number of walls and inclusion of pentagons-heptagons. The simplest form is the single-walled carbon nanotube (SWNT), which resembles a honeycomb graphite layer rolled into a monoatomic-thick cylinder. Several concentrically embedded SWNTs form a multi-walled carbon nanotube (MWNT). Other nanotube varieties include nanotube bundles or ropes, inter-tube junctions, nanotori and coiled nanotubes [2-5].

A brief analysis of the patent activity on carbon nanotube-based applications reveals that the major slots in the pie chart are occupied (in decreasing percentage order) by field emission, energy storage, composites, nanoelectronics, sensors and actuators. Judging by the exponential increase in the number of journal papers and patent applications per year [6], the technological potential of nanotubes is still far from being fully explored. Moreover, other applications are appended to this list every day. The fifth place of sensors and actuators can be explained by their relative later invention [7, 8]. CNT-based sensors will undoubtedly climb into the top of the applications list.

In this dynamic context, the discovery and subsequent understanding of elementary physical mechanisms, supported by modeling and simulation, will be the key for custom-designed CNT-based devices for the next decade. In order to bridge micro-, meso- and macro-scales, as typically dictated by nanotube physics, it is desirable to find a hierarchy of models, for both ease of computation and conceptual understanding. Once models bridging different scales have been worked out, it will become possible to analyze and optimize materials properties at different levels of approximation, eventually leading to the theoretical understanding of materials or even design of novel forms. A further step will consist in constructing predictive models, going beyond the simulation of fundamental properties, that will assist the innovation and design of CNT-based devices.

Investigating nanotube properties through modeling and simulation is preferable in a context in which nanotube manipulation and characterization are still limited, time consuming and expensive. This does not automatically mean that one has to accept blindly the validity of any calculations, at least within the gross approximations sometimes employed. It is precisely at this point that theoretical models and measurements have to be brought together and re-aligned. Higher order corrections, validity ranges and hints for model improvements are obtained in this way.

Due to their relative simplicity and atomically precise morphology, SWNTs offer the opportunity of assessing the validity of different macro- and microscopic 
models. For this reason, SWNTs will receive special attention in this chapter. Encouragingly, for SWNTs, the agreement between theoretical predictions and experimental data is constantly improving. As a side note, it is actually via simple theoretical calculations that the metallic/semiconducting nature of nanotubes, their Young's moduli and optical transitions were predicted in the first place.

The aim of this chapter is to give a brief introduction to CNT-based sensing from a modeling and simulation perspective. The remainder of this chapter is split into four main sections. Section 5.2 gives a quick overview of the basic properties of CNTs, with emphasis on the tight-binding band structure and density of states. A condensed state-of-the-art of experimental measurements on CNT sensor demonstrators is given in Section 5.3. In Section 5.4, we list the common modeling methods for calculations of the electronic structure, transport and mechanical properties calculations of the CNT-based devices. A number of illustrative modeling case studies are presented in Section 5.5, with the goal of showing how the often difficult task of modeling CNT systems should be tackled.

\section{2}

\section{Properties of Carbon Nanotubes: Reminder}

The purpose of this section is to provide a brief survey of the properties of carbon nanotubes necessary to understand the different sensing mechanisms analyzed in the next section. A review of the tools available for modeling the properties of CNTs will be developed later in Section 5.4. For a thorough analysis of the properties of nanotubes we refer the reader to any of the many excellent books and review articles available on this subject [2, 3, 9].

Broadly speaking, the properties of carbon nanotubes ca be grouped in three categories: structural, mechanical and electronic. From the structural point of view, in most situations CNTs can be considered one-dimensional (1D) objects, with typical diameters $\left(d_{t}\right)$ in the nanometer range and lengths $(L)$ reaching several micrometers. This one-dimensionality of tubes impacts on and is visible mostly through the mechanical and electronic properties. However, the structure of nanotubes can be exploited in itself such as for instance by field emitters or gas breakdown sensors, which are based on the "sharpness" of CNTs giving rise to huge local electric fields.

The mechanical properties class encompasses the elastic, thermal, vibrational or any other properties related to the motion of the tube's atoms. In nanotubes, carbon is $\mathrm{sp}^{2}$-hybridized, resulting in strong $\sigma$-bonds weakly reinforced by $\pi$-bonds. Considering the hybridization, it is natural to assume a certain overlap between nanotube and graphite (graphene) elastic properties, such as Young's modulus, bending, tensile and torsional stiffness and yield strength. SWNTs have tensile moduli close to $1 \mathrm{TPa}$ (stiff as diamond) and strengths of $\sim 50 \mathrm{GPa}$ (corresponding to $5-10 \%$ maximal strain), which earned them the title of ultimate fibers. Despite their stiffness, CNTs retain a high bending flexibility due to their high aspect ratios $\left(L / d_{\mathrm{t}}\right)$. With some exceptions, the thermal and vibrational properties of nanotubes 
also show similarities with graphite. Since the in-plane thermal conductivity of pyrolytic graphite is very high, it is expected that the on-axis thermal conductivity of defect-free tubes would be even higher. At low temperatures the phonon mean free path is controlled mainly by boundary scattering and the coherence length (micrometer scale) is larger in tubules than in high oriented pyrolytic graphite $(<0.1 \mu \mathrm{m})$. Another difference between CNTs and graphite is the Raman spectra, exhibiting new signal features in tubes, particularly the radial breathing mode

2 (RBM), specific to a cylindrical geometry (see Chapter $\bullet \cdot$ ).

Since it refers to the response of the fermion system to different excitations, the class of electronic properties practically encompasses all remaining properties. Therefore, it contains transport and electric properties (classical, spin-dependent and superconductivity, dielectric permittivity), optical properties (absorption, scattering, luminescence), magnetic properties (susceptibility, Zeeman splitting, Aharonov-Bohm effect), chemical properties (covalent and noncovalent binding), in addition to hybrid properties and correlated many-body effects (thermopower, piezoresistivity, piezoelectricity, Coulomb blockade, Kondo effect, TomonagaLuttinger liquid behavior).

In this chapter, we touch on only basic properties and include, whenever possible, references to texts treating more advanced topics. We indicate that a simple zone folding of the graphitic tight-binding (TB) band structure is able to explain most of these basic electronic properties, provided that the diameter tube $d_{\mathrm{t}}$ is large enough. For small nanotubes $\left(d_{\mathrm{t}}<12 \AA\right)$ curvature induces hybridization between of the $\pi$ and $\sigma$ bands of graphite changing the electronic band structure. Henceforth, we exploit the TB description, and return later to more refined models such as ab initio density functional theory (see Section 5.4).

The electronic properties of nanotubes are strongly modulated by small structural variations; in particular, their metallic or semiconducting character is determined by the diameter and helicity (chirality) of the carbon atoms in the tube. To understand this dependence, one has to start from the energy dispersion relations of graphene in the vicinity of the Fermi level. A major simplification is achieved by noting that only $\pi-\pi^{*}$ bands populate this spectral region, and $\sigma$-bands can therefore be removed with almost no influence. Subsequently, in going from the graphene to a nanotube, periodic boundary conditions for the wavefunctions along the circumference are imposed (zone folding), resulting in the quantization of the wavevector component along this direction. The derivation details of the tightbinding graphene dispersion relations and the subsequent zone folding procedure can be found in Reference [2]. As an example, for armchair metallic tubes of chirality $(n, n)$, the analytically derived bands are given by

$$
E_{q}^{ \pm}(k)= \pm \gamma_{0} \sqrt{1 \pm 4 \cos \frac{k a}{2} \cos \frac{q \pi}{N}+4 \cos ^{2} \frac{k a}{2}}
$$

where $q(\in \overline{1,2 N})$ specifies the discrete part of the wavevector perpendicular to the tube axis (i.e. the band index), $k$ is the continuous component that describes eigenstates in a given subband $(-\pi<k a<\pi), a=2.46 \AA$ is the graphene lattice 

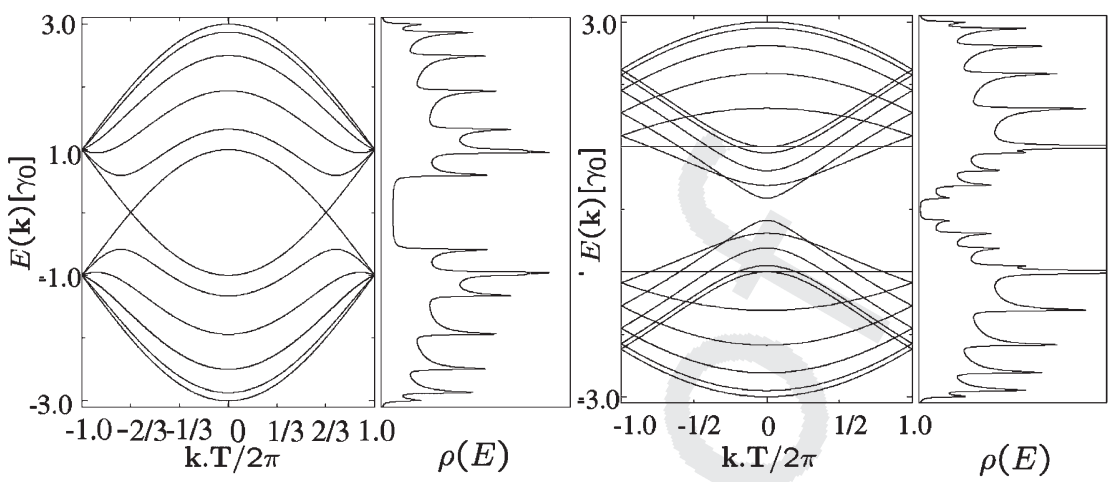

Figure 5.1 Dispersion relations and density of states for the metallic $(5,5)$ and semiconducting $(10,0)$ nanotubes.

constant and $\gamma_{0} \approx 2.9 \mathrm{eV}^{1)}$ is the hopping integral matrix element between nearestneighbor atoms.

As a matter of illustration, the band structures and densities of states (DoS) for the metallic $(5,5)$ armchair tube and the semiconducting $(10,0)$ zigzag tube are shown in Figure 5.1. Densities of states are calculated by tracing the spectral measure operator $\delta(E-\hat{H})$ over the band index and first Brillouin zone, i.e.

$$
\rho(E)=\sum_{q} \int \mathrm{d} k\left|\partial E_{q}(k) / \partial k\right|^{-1} \delta\left[E-E_{q}(k)\right]
$$

The intrinsic one-dimensionality of the CNTs gives rise to a number of sharp features $(\rightarrow \infty)$ in the DoS (see Figure 5.1) called van Hove singularities (vHS). The vHS positions are derived from the condition $\partial E_{q}(k) / \partial k=0$, which yields the solutions $\varepsilon_{q}= \pm \gamma_{0} \sin (q \pi / N)$. Because of these singularities, optical transitions are resonantly enhanced at energies corresponding to $2 \gamma_{0} \sin (q \pi / N)$.

For metallic SWNTs, the development of the dispersion relations to first order at the Fermi level yields a Fermi velocity $v_{F} \approx 10^{6} \mathrm{~m} \mathrm{~s}^{-1}$, independent of chirality. Thus, in the absence of electron scattering, the intrinsic conductance of a metallic tube should be $2 G_{0}$, where $G_{0}=2 e^{2} / h=(1 / 12.906) \mathrm{k} \Omega^{-1}$ is the conductance quantum, because there are two open channels at the Fermi level, each with a conductance $G_{0}$. On the other hand, semiconducting SWNTs have a bandgap $E_{g} \approx 0.9 / d_{\mathrm{t}}[\mathrm{eV}]^{2)}$, with $d_{\mathrm{t}}$ the nanotube diameter in nanometers. The next section will review how these remarkable properties have resulted in experimental breakthrough sensor demonstrators.

1) The actual value of the $\gamma_{0}$ parameter depends on the property to be analyzed; e.g. the value for STM is $\sim 2.5 \mathrm{eV}$ whereas that used in Raman spectroscopy $\in 2.9-3.1 \mathrm{eV}$.

Reference [47] and references therein). Also the dispersion becomes nonlinear for large tubes, known as the trigonal wrapping effect (see Chapter •.). Although important for excitations,

2) Here the gap refers to the quasi-particle gap, which is different from the optical gap relevant for Raman spectroscopy (see

these effects do not affect too much the transport properties close to $\mathrm{E}_{\mathrm{F}}$. 
5.3

Carbon Nanotube-Based Sensor Demonstrators: State-of-the-Art

Continuously expanding, the field of CNT-based sensors includes an already impressive list of demonstrators, encompassing (bio)chemical, strain, stress, pressure, mass, flow, thermal and optical sensors. The field is too vast to be surveyed exhaustively here and is also not the focus of this chapter dealing with modeling CNT sensing devices ${ }^{3)}$. Rather, the goal of this concise state-ofthe-art is to present to the reader a survey of possible sensing mechanisms involved so far in CNT sensors. After this section, it should become clear what kind of modeling tools are necessary to investigate nanotube devices. In analogy with the classification made in the previous section, we group sensing mechanisms in three categories, centered around the electronic and the mechanical properties, plus other mechanisms.

\subsection{1}

\section{Modulation of Electronic Properties}

In terms of number of publications, sensors involving the modulation of a CNT's electronic properties lead the competition by far. This can be easily understood by a brief examination of the structure of a nanotube. A CNT is hollow, meaning that only "surface" electronic states are present, making a tube extremely sensitive to any perturbations.

It is precisely this observation that led Kong et al. [7] and Collins et al. [8] to propose CNTs as sensitive materials for chemical sensors. Kong et al. [7] found the electrical conductance of a nanotube to increase when the tube was exposed to $\mathrm{NO}_{2}$, and to decrease when exposed to $\mathrm{NH}_{3}$. Electron charge transfer (doping) was proposed as the mechanism dictating the change in conductivity $(\Delta G)$ by shifting the Fermi level of the channel. Collins et al. showned [8] that exposure to air or oxygen dramatically influences the nanotubes' electrical resistance, thermoelectric power and local density of states, as determined by transport measurements and scanning tunneling spectroscopy. These electronic parameters can be reversibly "tuned" by surprisingly small concentrations of adsorbed gases, and semiconducting nanotubes can apparently be converted into metallic tubes through such exposure. Since these initial two reports, the list of chemFET-like gas and biological sensors has increased considerably ${ }^{4}$. Innovations have also been brought, such as nanotube functionalization, which has yielded highly sensitive and selective sensors [12, 13]. Also, Goldsmith et al. [14] have recently demonstrated that it is possible to control the functionalization density by monitoring in real time the conductance of a nanotube in an electrochemical setup.

Carbon nanotube-based chemFETs deserve special attention. Although they were the first type of sensors proposed, the understanding of their operation is still hindered by the multitude of potential effects stemming from the interaction

4) See Table 1 in Reference [10] for a list of detected agents so far. 
of chemical molecules with this system. Among these, molecules interacting with chemFETs can: (i) if charged and isolated from nanotube, gate the transistor (field effect), (ii) if polar, modify the gate capacitance, (iii) regardless of charge, dope the nanotube or modify the work function of the contacting metal resulting in a Schottky barrier modulation, or (iv) increase carrier scattering. All these mechanisms overlap, rendering it very difficult experimentally to tell which one is responsible for the $I-V$ characteristic. This motivates the development of modeling tools capable, in principle, of identifying the correct mechanism.

Another emergent sub-family of chemical sensors exploit subtle changes in the polarizability of nanotubes subject to gas exposure, which can result in either capacitance modulation $\Delta C$, as shown by Snow et al. [15], or in a shift of the electrical resonant frequency of a microwave circuit $\Delta f_{0}$, as demonstrated by Chopra et al. [16]. As suggested by Snow et al. [11], future generation chemical SWNT sensors could exploit simultaneously $\Delta G$ (charge transfer) and $\Delta C$ (dipole moments and polarizability), increasing the information gathered from a single device with potential improvements in resolution, sensitivity and selectivity.

It is also worth mentioning that reading out the modulation of the electronic properties in chemical sensors needs not be confined to electrical measurements. For example, Sumanasekera et al. [17] have utilized thermopower measurements (see also Reference [8]) to evidence the sensitivity of CNTs to $\mathrm{C}_{6} \mathrm{H}_{6}$. An even more promising approach is using light to probe either the photo-absorption or the fluorescence spectra [18, 19]. Cao et al. [18] have studied band-gap photo-absorption in SWNTs, previously exposed to air, in different organic solvents. The observed bleach and recovery of optical spectra are due to the charge transfer between nanotubes and adsorbates (e.g. $\mathrm{H}_{2} \mathrm{O}$ ), in their process of (ad)/(de)sorption from the nanotube surface. Heller et al. [19] have shown that DNA-wrapped SWNTs can be placed inside living cells to detect trace amounts of harmful contaminants using near-infrared light. When the ensemble is exposed to certain ions $\mathrm{CHg}^{2+}, \mathrm{Co}^{2+}, \mathrm{Ca}^{2+}$, $\mathrm{Mg}^{2+}$ ) the wrapping DNA changes shape from the B form to $\mathrm{Z}$ form, reducing the surface area covered by the DNA, which further perturbs the electronic structure and shifts the nanotube's natural, near-IR fluorescence to a lower energy. These results open the door to new types of optical sensors and biomarkers inside living systems (in vivo).

The second best known family of CNT-based sensors is the one exploiting the huge piezoresistive gauge factors reported for nanotubes. We do not insist on this

5 family, since Chapter $\cdot \bullet$ is dedicated entirely to this subject. We only mention that piezoresistive pressure, force (stress) and strain sensors utilize the band-gap modulation of nanotubes subject to torsional or tensile strain. The effect should be

6 visible either via transport or via Raman spectra (see Chapter $\bullet \cdot$ ).

\section{3 .2}

\section{Shifting of Mechanical Resonances}

There are not many reports of sensors in this category, but we discuss resonant CNT devices, motivated by their potential in sensing applications. Due to the huge 
stiffness and extremely low mass density - carbon is one of the lightest atoms, and SWNTs are one atom thick - nanotubes hold the promise of unequaled high resonating frequencies and mass sensitivities.

The first to propose CNT as nanobalances were Poncharal et al. [20], demonstrating femtogram-resolution mass detection with large MWNT cantilevers. A transmission electron microscope (TEM) was utilized in tracking the resonant frequency. Later, by moving the setup inside a scanning electron microscope (SEM), and analyzing secondary electron intensities, Nishio et al. [21] achieved zeptogram sensitivities. The measuring schemes in these references are obviously not suitable for large-scale integration. Nevertheless, recently, tunable CNT resonators have been reported [22-24], opening the way to integrated nanobalances for minute mass detection.

\section{3 .3}

\section{Other Transduction Mechanisms}

The list of CNT-based sensors is far from being closed, with exotic, new sensor mechanisms frequently being proposed. Here we provide a brief selection of promising devices that do not necessarily conform to the previous two subsections.

Ghosh et al. reported [25] flow sensors in which the conductance of carbon nanotubes, disposed parallel to the flow lines, is clearly changed at different flow rates. The suggested mechanism for this nonlinear effect is forcing of the electrons in the nanotubes by the fluctuating Coulombic field of the liquid, or, briefly, Coulomb drag. Another CNT flow meter demonstrated recently by Bourlon et al. [26] operates differently from the previous device. A CN-FET is placed within the electrical double layer of an $\mathrm{SiO}_{2}$ channel (i.e. almost tangentially with one of the internal surface of the channel), being gated by the flow rate-dependent $\zeta$-potential.

An ionization CNT sensor has been demonstrated by Modi et al. [27], based on the ionization fingerprint which is characteristic to each analyte gas. This device simply exploits the geometry of the nanotubes, since their sharp tips generate very high electric fields at relatively low voltages, lowering gas breakdown voltages. Another category of sensor demonstrators that make use of CNTs as electrodes are the amperometric biosensors utilizing nanotubes as electrode material in electrochemical setups. This type of sensor is exhaustively reviewed in Reference [28], and will not be analyzed here.

Still other CNT sensing devices could be mentioned here, such as low-temperature quantum electrometers [29] and superconducting quantum interference device (SQUID) magnetometers [30]. Nevertheless, understanding the physics of these devices is beyond the scope of this chapter. We therefore end this section here, and reassert that CNT sensors are based on a huge variety of sensing mechanisms. Although unifying principles exist, which have allowed us, for instance, to group the sensors in three major classes, the spectrum of phenomena is still too broad to be addressed by a single modeling methodology. The following section 
will attempt to display the tool available for the modeling of nanotubes, trying at the same time to identify their context-dependent usefulness and limitations.

\section{4 \\ Modeling: Reviewing Methods and Tools}

Because of their nanometric diameters, carbon nanotubes are quantum objects, and are therefore governed by the many-body Schrödinger equation. The Schrödinger equation is still unsolved for systems more complex than the hydrogen atom, forcing series of approximations followed by numerical simulation. The most frequent approximations utilized in solid-state physics and quantum chemistry include mean-field (or effective) electronic structure theories (tight binding, Hartree-Fock, density functional theory), within the Born-Oppenheimer approximation that decouples the motion of atoms from the electronic structure problem. This is also the dominant workbench in nanotube science.

Even within the simplest framework, the large number of atoms involved in a typical CNT-based nanodevice is a major bottleneck. The recurrent problem in nanotube modeling is a certain square (Hamiltonian diagonalization or Green's functions calculations) or cubic scaling (inter-atomic force calculation for molecular dynamics) in the number of atoms, which drives simulation into the intractable realm. Sometimes, when translational symmetry is lost, simulations might involve $10^{5}$ atoms or even more, particularly in the modeling of transport within chemically perturbed tubes or vibrational properties of long nanotube cantilevers (see Section 5.5). Storing a matrix with $10^{5} \times 10^{5}=10^{10}$ elements is costly but this is nothing compared with the time it would take to invert or diagonalize such a matrix, requiring around $\left(10^{5}\right)^{3}$ operations.

In the light of computational challenges engendered by modeling CNT-based devices, we start with a brief review of the different approaches and methods available for the task of modeling both properties and devices based on carbon nanotubes.

\section{4 .1}

\section{Electronic Structure Calculations}

The strong similarity of the short-range chemical order of carbon nanotubes to that of graphite $[31,32]$ allows theoretical analyses based on empirical methodologies imported from graphite. The methodology spectrum is wide and ranges from the direct zone folding of the graphite results to the quantum-mechanical tightbinding Hamiltonians fitted to graphite properties. The performance of the different techniques varies, from the qualitative picture offered by zone folding, with intrinsic deficiencies at low frequencies, to the almost quantitative results of tightbinding approaches.

Zone folding and tight binding are at the base of the very first electronic structure predictions for carbon nanotubes, partly discussed in Section 5.2. Electronic 
properties in the vicinity of the Fermi level have been successfully captured for single- and multi-walled nanotubes, bundles and even topological defects [3, 33, 34], with the simplest $\pi$-band nearest-neighbor tight-binding Hamiltonian ${ }^{5}$

$$
\hat{H}=\sum_{v} \varepsilon_{0}|v\rangle\left\langle v\left|+\sum_{v} \sum_{\mu \in \mathrm{nb}(v)} \gamma_{0}\right| v\right\rangle\langle\mu|
$$

where $\varepsilon_{0}$ is the on-site energy (typical value 0 , that shifts the Fermi level to $E_{F}=$ $0), \gamma_{0}$ the hopping integral (typical value $\left.-2.7 \mathrm{eV}^{2}\right),|v\rangle$ and $|\mu\rangle$ are $\mathrm{p}_{z}$ orbitals located at different atomic positions and $\mathrm{nb}(v)$ is a function giving the nearest topological neighbors of $|v\rangle^{6}$. The first limitation of this model is revealed by systems involving charge transfer, such as charge redistribution at the interface between a CNT and a metallic contact in a solid-state device, or the interaction of a tube with a molecule. Although attempts in developing charge self-consistency to tight-binding calculations have been made [35], in a situation involving strong transfer (or rehybridization) one has to be cautious in employing these schemes. The only solution to this is ab initio electronic structure methods.

First principles ( $a b$ initio) total energy calculations are one of the most accurate methods available to the study of nanotubes. These calculations provide the bonding, electronic structure and atomic arrangement, and are nowadays based on density functional theory (DFT) [36]. For a general description of DFT and its applications, see Reference [37]. This allows the calculation of the ground-state and dynamic properties of a many-electron system from a simple one-electron, effective Schrödinger equation (a mean-field approximation). In this theory, the total energy, expressed as a functional of the total electron density $\rho(\mathbf{r})$, is decomposed into three contributions. These are the well-known kinetic energy term of non-interacting particles, the Coulomb energy due to classical electrostatic interactions and a part that takes care of the many-electron interactions, so-called exchange-correlation energy. The effective one-electron eigenfunctions and eigenvalues allow for a formulation of bonding and structure in terms of molecular orbitals and band structure of solids. The fact that the problem has been reformulated in terms of independent electrons does not mean that correlations are ignored. This formulation is based on a self-consistent procedure in which the effective potential depends on the electron density that also depends on the oneelectron eigenfunctions. The achievement of self-consistency is one of the main technical problems in first-principles calculations [38], raising the complexity to $\mathcal{O}\left(N^{3}\right)$, with $N$ the number of atoms.

When still more accurate results, or non-ground-state properties (e.g. electronic excitations) are required, many-body electronic correlations are explicitly included. Depending on the origin field, correlations are added as in quantum chemistry via

5) This Hamiltonian can only describe single tube properties. Intertube coupling terms are necessary to model multi-walled or tube bundles. Examples of such Hamiltonians are given in Section 5.5.2.4 and Section 5.5.2.5.

6) Hereafter, the summation limit $\mu \in \mathrm{nb}(v)$ will be replaced by $\mu \neq v$. 
so-called post-Hartree-Fock methods such as configuration interaction (CI), Møller-Plesset (MP) or coupled-cluster (CC) [39], or as in condensed-matter physics via perturbation theory supported by diagrammatic techniques [40-42]. Regardless of the choice, the numerical complexity is boosted to at least $\mathcal{O}\left(N^{6}\right)$. An example of where these methods are required is the accurate calculation of optical spectra in carbon nanotubes, known to be modified from the simple vHS as obtained via tight binding (see Section 5.2) by excitonic effects. The optics of nanotubes is of outmost importance as it offers one of the few reliable frameworks

7 for CNT characterization (e.g. Raman spectroscopy, Chapter ••). The most widely used methods to include excitonic effects are time-dependent density functional theory (TDDFT) [43] and the GW scheme followed by the Bethe-Salpeter equation (BSE) solution [44]. Also applied to carbon nanotubes [45] (TDDFT [46]; GW+BSE [47]), these many-body corrections have shown a major deviation from the simple tight-binding picture, with optical transitions modified by as much as $\sim 0.5 \mathrm{eV}$. We do not continue detailing many-body methods hereafter and refer the reader to the previous citations for thorough treatments. We do mention, however, that other electron-correlation effects might be present in CNTs, especially at low temperature, including Tomonaga-Luttinger liquid behavior, Coulomb blockade, the Kondo effect and superconducting contacts [3]. Another important effect not treated in this chapter is the electron-phonon interaction that leads to a meanfree-path reduction, and therefore plays a major role in quantum transport in nanotubes.

\section{4 .2}

Transport Formalisms

The electronic transport properties of nanostructures are governed by the quantum conductance scaling features, that range from ballistic to diffusive or localized regimes, depending on the strength of quantum interference effects and decoherence phenomena. As device geometries are downsized to the ultimate limits of miniaturization, and become comparable to the elastic mean free path for the electrons, the transport enters the ballistic regime, which is characterized by vanishingly low intrinsic dissipation.

In Section 5.3.1 we gave a brief glimpse of a number of ways in which a molecule, for instance, can interact with a chemFET device. This example is very appropriate since it allows us to assess the typical challenges encountered in modeling CNT-based sensors. To determine to what extent the observed currentvoltage $(I-V)$ device characteristic can be attributed to the adsorption of analyte molecules, several tasks have to be tackled. First, the interaction between CNT and attached molecules, and resulting chemical binding energies, charge transfer and polarizability need to be precisely described from first principles approaches. The role of topological defects along the SWNT sidewall should be analyzed owing to their particular chemical reactivity level, which might severely affect the sorption properties of other chemical vapors. The consequent impact of this functionaliza- 
tion on the resulting intrinsic charge transport properties need then to be assessed initially for quantum coherent regimes, but also for out-of equilibrium situations, in which electrostatics and intrinsic dissipation (e.g. electron-phonon interaction) might play a substantial role. Finally, the changes in the dipole properties at the metal/CNT interfaces must be investigated to explore the potential modulations of charge injection through the resulting modified Schottky barrier.

Anantram and Léonard [48] reviewed the physics and modeling of carbon nanotubebased field-effect transistors (CNFETs), focusing on issues related to electrostatics and Schottky barriers. There is, however, no straightforward prescription on how to extend the presented methods to chemical sensors.

Tools developed for modeling molecular electronics devices can in principle also be applied to nanotubes. The main approach nowadays is a methodology combining non-equilibrium Green's functions (NEGF) with density functional theory (DFT) [49]. Several surveys and books are available for molecular transport [50, 51]. It is also noteworthy that NEGF can be combined with more advanced electronic properties schemes for a more accurate description of correlations, such as the NEGF-GW approach detailed in Reference [52]. However, CNTs are far bigger than common molecular electronics devices. A self-consistent, linearly scaling (order- $N$ ) transport method for nanotube device simulation is still missing ${ }^{7}$. Therefore, both in the past and currently, one has to rely on assumptions about the CNT-metal contacts, electrostatics and charge transfer with chemical species. The utilized model Hamiltonian becomes the central object, crucially influencing the results obtained. Hamiltonian choices and/or parameterizations are a recurrent theme in Section 5.5.2.

Once a Hamiltonian model has been obtained, investigating coherent quantum transport in a nanotube segment of length $L$ with reflectionless contacts to external reservoirs is achieved in practice mainly via two complementary transport formalisms ${ }^{8}$. First, following linear response theory, the Kubo conductance is computed as $G(E)=\left(2 e^{2} / L\right) \lim _{t \rightarrow \infty} \operatorname{Tr}[\hat{D}(t) \delta(E-\hat{H})]$, where $\Delta(E-\hat{H})$ is the spectral measure operator, whose trace gives the total density of states (DoS). $\hat{D}(t)$ is the diffusivity operator defined as $\hat{D}(t)=\left(\hat{X}(t)-\hat{X}(0)^{2} / t\right.$ with the help of $\hat{X}(t)$, the Heisenberg representation of the position operator [53]. Much information about the propagation of electrons can be gained from the energy-dependent diffusion coefficient, the ensemble average of $\hat{D}(t)$ :

$$
D(E, t)=\frac{\operatorname{Tr}\left[(X(t)-X(0))^{2} \delta(E-H)\right]}{t \operatorname{Tr}[\delta(E-H)]}
$$

In the presence of static disorder, the time-dependent diffusivity for a given wave packet always reaches a saturation regime, whose value will be related to the

7) Self-consistency is highlighted because this affirmation is valid only for this case. Once the charge transfer problem has been solved, with a self-consistent charge and potential distribution along the device, transmission or diffusion coefficients can be calculated using order- $N$ methods (see below).

8) Unless explicitly specified, the temperature is assumed to be $0 \mathrm{~K}$ in all transport calculations. 
elastic mean free path $\ell_{\mathrm{e}}(E)$ through $D(E) \propto v(E) \ell_{\mathrm{e}}(E)$, with $v(E)$ the velocity of a wave packet of energy $E$. Such an approach, implemented using order- $N[\mathcal{O}(N)]$ computational techniques, has been successfully compared to analytical results derived from the Fermi Golden Rule (FGR), but for uniform disorder [53-55] (see Section 5.5.2.1). It has also been extended to more realistic disorder models such as chemical substitutions (Section 5.5.2.2) and physisorbed molecules (Section 5.5.2.4).

In another approach, the Landauer-Büttiker conductance is evaluated from the transmission coefficient $G(E)=\left(2 e^{2} / h\right) T(E)$. In a multi-terminal setup in which the leads are labeled by $\lambda$, the transmission coefficients between any two contacts ${ }^{9)}$ is given by

$$
T_{\lambda \lambda^{\prime}}(E)=\operatorname{Tr}\left[\Gamma_{\lambda}(E) G^{(r)}(E) \Gamma_{\lambda^{\prime}}(E) G^{(a)}(E)\right]
$$

in terms of the retarded Green's function $\hat{G}^{(r)}(E)=\left[E \hat{I}-\hat{H}-\Sigma_{\lambda} \hat{\Sigma}_{\lambda}(E)\right]^{-1}\left[\hat{G}^{(a)}(E)=\right.$ $\left.\hat{G}^{(r) \dagger}(E)\right]$ and level broadening functions (escape rates) $\hat{\Gamma}_{\lambda}(E)$ given by $i\left[\Sigma_{\lambda}(E)-\Sigma_{\lambda}(E)\right] . \hat{\Sigma}_{\lambda}(E)$ stands for the self-energy accounting for the coupling with the lead- $\lambda$ [50]. In general, order- $N$ methods to evaluate these quantities are facilitated by the 1D character of CNTs. For instance, $\hat{\Sigma}_{\lambda}(E)$ are typically obtained through the decimation technique [56,57], while $\hat{G}^{(r)}(E)$ can be obtained either by tube slicing and fast elimination methods [57, 58], or by two-sided Lanczos recursion [59]. At times, it is also useful to calculate the current through lead- $\lambda$, which is achieved by integrating in energy and summing over $\lambda^{\prime} \neq \lambda$ :

$$
I_{\lambda}=\sum_{\lambda^{\prime} \neq \lambda} \frac{2 e}{h} \int_{-\infty}^{\infty} T_{\lambda \lambda^{\prime}}(E)\left[f\left(E, \mu_{\lambda}\right)-f\left(E, \mu_{\lambda^{\prime}}\right)\right] \mathrm{d} E
$$

in which $f\left(E, \mu_{\lambda}\right)=1 /\left[1+e^{\left(E-\mu_{\lambda}\right) / k_{\mathrm{B}} T}\right]$ is simply the Fermi-Dirac distribution of states in lead- $\lambda$, considered in thermal equilibrium and having the chemical potential $\mu_{\lambda}$.

A connection between the Landauer and Kubo conductances is made possible through the ergodic assumption [60], i.e. $\bar{T}=T=N_{\perp}(E) \ell_{\mathrm{e}}(E) / L$, where $\bar{T}$ is the averaged transmission coefficient. To ensure convergence of $\bar{T}$, averaging over a few hundred configurations of disorder is performed in practice.

\subsection{3}

\section{Mechanical Models}

The classical continuum beam theory, although questionable for nanometric objects, has produced the first predictions of nanotube elastic properties, and is still employed nowadays with relatively good results [2, 61]. This theory contains

9) Assuming reflectionless contacts, the terms leads, terminals and contacts can be used interchangeably (see Reference [50] for a thorough discussion). 
a parameter, the Young's modulus, which still proves to be extremely hard to measure experimentally. Therefore, simplified analytical models with periodic boundary conditions were initially used for evaluating the Young's modulus of nanotubes and graphene, utilizing for instance the Tersoff-Brenner potential [62]. Later, molecular dynamics (MD) imposed itself as the common choice allowing the simulation of finite systems composed of nanotubes in a variety of configurations.

There is currently a large variety of force-fields to drive MD simulations. For carbon-based systems, analytic many-body force-fields such as Tersoff-Brenner and Stillinger-Weber have long been available [62]. The Tersoff-Brenner potential works particularly well for crystalline, amorphous and molecular phases of carbon, such as diamond, graphite, fullerenes and nanotubes and has been thoroughly tested in a variety of settings. An important bonus for the Tersoff-Brenner potential is that it is reactive, i.e. chemical bonds can form and break during the simulation. Consequently, the neighbor list of each atom is dynamic that slows the simulation to a certain extent. Therefore, for larger systems it is often convenient to turn to simpler, fixed-topology force-fields such as CHARMM or Amber [63].

More realistic models including electronic effects are obtained through tightbinding methods [64] using a minimum sp-basis. The TB approximation captures part of the chemical strain through the geometry dependence of its electronic matrix elements. However, when still higher accuracy is desired, ab initio methods can be used.

$A b$ initio molecular dynamics simulations based on DFT can be performed by minimizing the total energy with respect to the eigenfunctions for a given ionic configuration, then computing the forces and moving the ions [65]. A different and very elegant scheme was proposed by Carn and Parrinello [66]. The idea is to introduce a fictitious electronic dynamics, which keeps, during the ionic motion, the electronic wavefunction adiabatically close to the instantaneous eigenstates of the quantum Hamiltonian. In this approach, both the ions and the eigenfunctions are treated as classical fields following the Newtonian dynamics, with the orbitals subject to the constraint of orthonormality. The trade-off in the simulations is between having smaller electronic masses, thus keeping the system close to the Born-Oppenheimer minimum, and the necessity of keeping the integration time step as large as possible, in order to simulate the dynamics for a time adequate for the atomic scales (typically from a fraction of a picosecond up to several picoseconds). This technique can be used both for molecular dynamics simulations and for energy minimization of the DFT functional.

Finally, we draw attention to a promising use of model potentials; hybrid modeling. In situations where a large system contains a site of specific interest, one may succeed by treating the region of interest quantum mechanically and the rest with a model potential, accelerating significantly the computation time. The same technique can be generalized in replacing mildly bent nanotube segments by continuum beams (see Chapter $\bullet$ and references therein for details about hybrid modeling). 
5.5

Modeling Case Studies: Highlighting Physical Mechanisms

In the previous section we have briefly reviewed some of the most utilized modeling methods for carbon nanotubes. This final section will bring to attention a few modeling examples with the goal of illustrating the challenges but also the achievements of CNT-based systems simulation.

Some results coming from scanning tunneling microscopy simulations of nanotubes are first presented in the following subsection. Spectra for pristine CNTs are compared with spectra for nanotube ropes, CNT on Au substrates and CNTs with topologic Stone-Wales defects. This comparison will give an indication of the influence of different coupling on the electronic properties of nanotubes, and suits as a preparation for the following subsection, that will deal in detail with the interactions of CNTs with different molecules from a transport perspective. Section 5.5.2 will therefore dive into the transport phenomena in CNT and the effect of functionalization, doping, adsorption and other types of chemical disorder on these properties. From modeling properties, we move to a final example of a device-level modeling in Section 5.5.3. Both the mechanical and transport properties of an electromechanical deflection transducer are treated in this subsection with the goal of validating the device operation, and showing the usefulness of modeling tools in the design of future generation CNT-based devices.

\subsection{1}

\section{Scanning Tunneling Microscopy of Nanotubes}

Scanning tunneling microscopy (STM) and scanning tunneling spectroscopy (STS) are two extremely useful techniques in the study of the role of the local environment on the electronic properties of nanotubes. Therefore, this topic is relevant for sensors, in particular for those involving modulation of electronic properties (see Section 5.3.1). For a basic introduction into the simulation of STM images, see Reference [34] and references therein. Here we only discuss a selection of simulation results for CNTs in several configurations.

In the simplest approximation, STM topographic images can be obtained through the Tersoff-Hamann theory [67]. In this model, the tip is not taken into account explicitly, therefore convolution effects due to the tip shape are neglected. The STM current, for an external applied bias voltage $V$, is proportional to the local density of states (LDoS) integrated between the Fermi levels of the tip and sample:

$$
I(\mathbf{r}, V)=\int_{E_{\mathrm{F}}-V}^{E_{\mathrm{F}}} \mathrm{d} E \rho(\mathbf{r}, E)=\int_{E_{\mathrm{F}}-V}^{E_{\mathrm{F}}} \mathrm{d} E \sum_{i}\left|\psi_{i}(\mathbf{r})\right|^{2} \delta\left(E-E_{i}\right)
$$

where $\left\{\psi_{i}, E_{i}\right\}_{i}$ are electronic eigenfunctions and their corresponding eigenenergies. Formally, LDoS is nothing more than the diagonal matrix element of the 
spectral measure operator in real-space representation, $\rho(\mathbf{r}, E)=\langle\mathbf{r}|\delta(E-\hat{H})| \mathbf{r}\rangle$. The summation over $i$ can stand for summation over the band index $q$ and integration over the $k$ points of the first Brillouin zone of the tube in the infinite case (see Section 5.2), or a summation over the discrete energy level indices in the finite tube case. Both DFT Kohn-Sham or tight-binding (TB) Hamiltonian models can be used to obtain the electronic eigenfunctions and eigenenergies. Topographic images are subsequently approximated by iso-surfaces of $I(\mathbf{r}, V)$. On the other hand, in STS experiments, the accessed quantity is the differential conductance $\mathrm{d} I / \mathrm{d} V$, which within the above assumptions can be shown to be proportional to the nanotube density of states (DoS).

The first observation made from STM simulations is that the interaction with a substrate or with other tubes does not alter the STM patterns with respect to isolated tubes $[68,69]$. Finite length nanotubes exhibit standing wave patterns. In the case of armchair CNTs, the standing waves can be completely characterized by a set of four different three-dimensional shapes as catalogued in Figure 5.2. On a (111) gold substrate, even if the tubes are fairly strongly bond by charge transfer, the interaction with the substrate does not alter the main images of the isolated tubes (see Figure 5.3). The computed images are in very good agrement with the experiments in both wavelength of the standing wave $(0.75 \mathrm{~nm})$ and inner details (pairing). Surface states related to the boundary of the tubes are observed to appear within $1 \mathrm{eV}$ above the Fermi level.

We now focus on the modification of the scanning tunneling spectra by the tube-tube interactions. In Figure 5.4, calculated STM/STS images obtained for an external voltage of $+0.5 \mathrm{eV}$ for a bundle made of three $(8,8)$ nanotubes are compared with the isolated $(8,8)$ tube. Changing the polarity of the applied voltage does not introduce appreciable changes in the STM topographic image. However, the inter-tube interaction clearly modifies the spectra seen in the DoS. A "pseudo-gap" close to the Fermi level is opened, as predicted for random oriented nanotube ropes [70] (pseudo-gap $\approx 0.1 \mathrm{eV}$ ). The bundle remains metallic, however ${ }^{10)}$. Also, the electron-hole asymmetry in the DoS is accentuated and the spike structure of the van Hove singularities is smoothed out. The fact that the position in energy of the peaks is not strongly modified explains the success of using isolated SWNT spectra to describe the experimental data [71]. Nevertheless, the shape of the spectra (relative intensities) is strongly affected by tube-tube interactions, as is clearly seen in Figure 5.4 .

The role of topological defects, in particular the pentagon-heptagon pair and the Stone-Wales (SW) defect has also been investigated [34]. Typical results on a $(10,10)$ carbon nanotube for an applied bias potential of $\pm 1.5 \mathrm{eV}$ are presented in Figure 5.5. It can be observed that the SW defect creates a very localized modification of the image as compared with a perfect tube, although the decay length of the perturbation is very short $(\sim 1.5 \mathrm{~nm})$. The symmetry of the image corresponding to occupied/unoccupied states is completely different. Therefore,

10) These conclusions have been confirmed experimentally [72] for metallic tubes and ropes such as that shown here. 

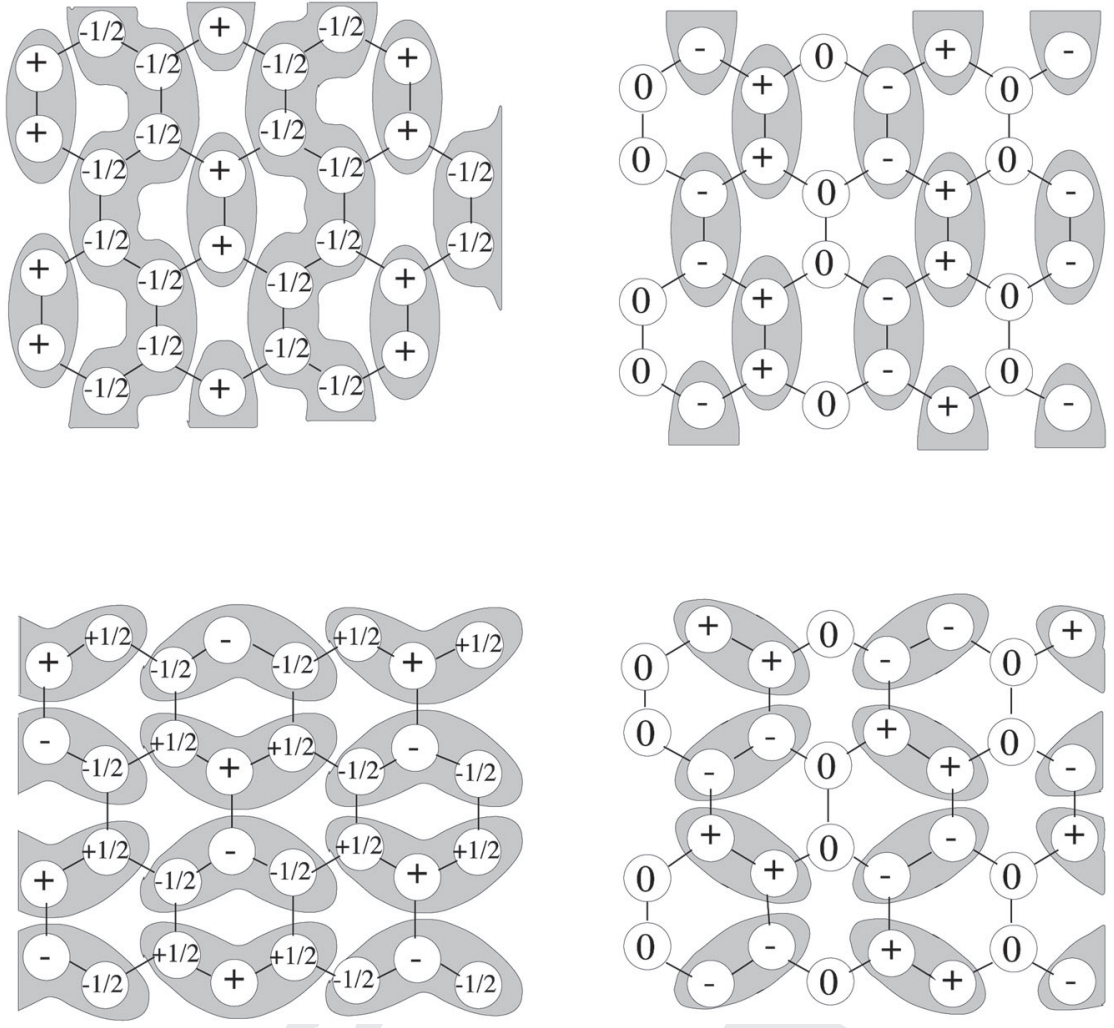

Figure 5.2 Schematic tight-binding catalog of STS images for an armchair nanotube close to the Fermi level, corresponding to the bonding and antibonding solutions in a 1D confinement box model (the wave-function values are indicated by the \pm symbols). The same scheme holds for supported tubes on a $\mathrm{Au}(111)$ substrate. Adapted from Reference [68].

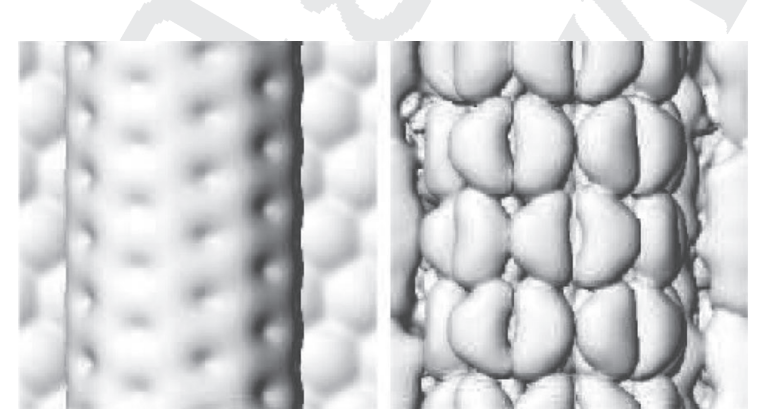

Figure 5.3 $A b$ initio calculation for a $(5,5)$ carbon nanotube supported on $\mathrm{Au}(111)$. Left: STM-topographic image for an applied voltage of $2 \mathrm{eV}$. Right: standing wave pattern of the highest occupied molecular orbital (HOMO). The latter pattern fits one of the catalog of STS images in Figure 5.2. Adapted from Reference [69]. 

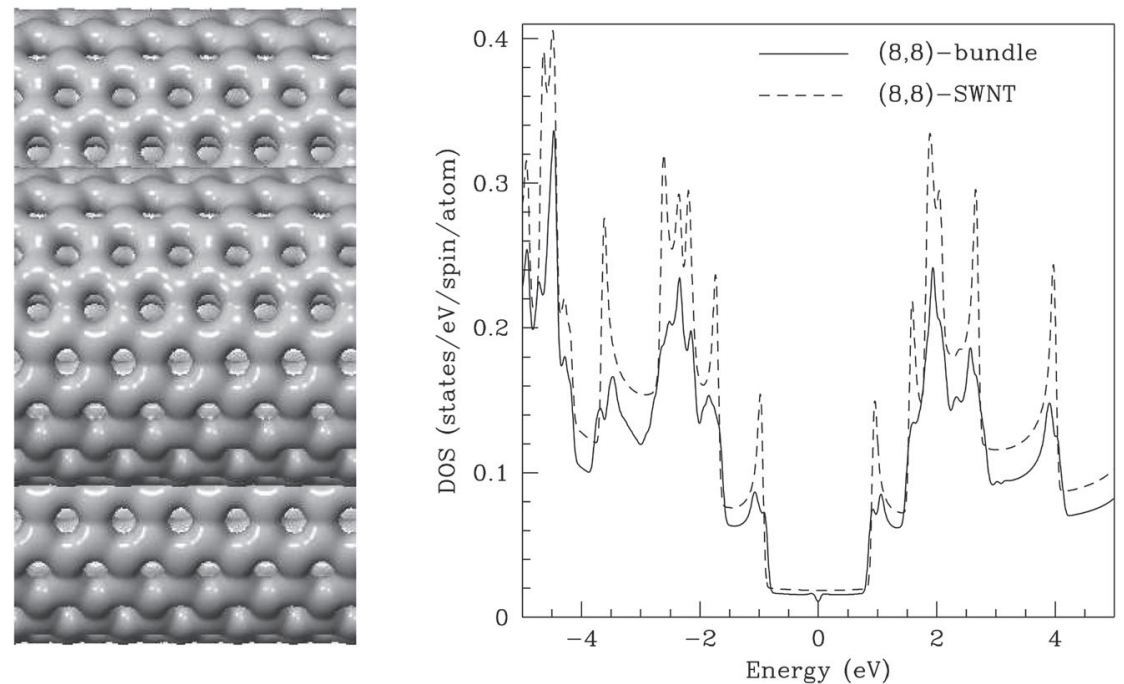

Figure 5.4 STM (a) and DoS (b) for a small CNT rope containing three $(8,8)$ SWNTs $\left(d_{\mathrm{t}}=1.09 \mathrm{~nm}\right)$ close-packed with an inter-tube distance of $0.345 \mathrm{~nm}$. The opening of a "pseudo-gap" of about $0.1 \mathrm{eV}$ around the Fermi level is clearly visible, by comparing the results for the bundle with the DoS of an isolated $(8,8)$ SWNT (dashed line). Adapted from Reference [69].

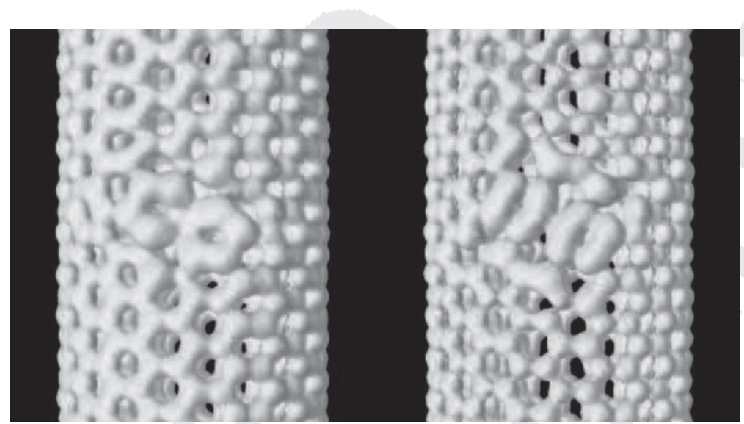

Figure 5.5 Simulated constant current STM images for a $(10,10)$ CNT with a single Stone-Wales (SW) defect for an applied external tip-sample bias of $\pm 1.5 \mathrm{eV}$. The orientation of the centered bond joining the two pentagons of the SW defect is set to $\pi / 4$ degrees to the tube axis.

these calculations indicate that SW defects could be experimentally accessible via STM measurements. Later in this section we will show that topological defects play a major role in influencing the conductance and chemical sensitivity of a carbon nanotube channel, and have to be addressed explicitly in CNT-based sensors. 
5.5 .2

Transport Phenomena in Carbon Nanotubes

The peculiar geometry of carbon nanotubes yields particularly strong quantum confinement, low-dimensional physics, but also enhanced sensitivity to any disruption of the $\mathrm{sp}^{2}$ network $[2,3,73]$. As emphasized in Section 5.3.1, this mechanism has fueled many experimental breakthroughs [7, 15, 74]. In this context, the development of efficient and predictive computational transport methodologies is fundamental in enabling in-depth exploration of complex hybrid nanotubes, with random distribution of functionalizing entities that will alter or modify the underlying carbon-based conducting channel properties [3].

Functional groups can specifically be attached to the carbon nanotube surface either by physisorption or by covalent bonding. Conventional covalent functionalization has been experimentally demonstrated to perturb severely the atomic structure of the CNT and its corresponding electronic properties. In contrast, the physisorption of organic molecules on the nanotube sidewalls is an example of noncovalent functionalization involving $\pi$-stacking interactions and corresponding to a much weaker binding energy. The main interest in noncovalent functionalization also stems from the negligible charge transfer involved within the $\pi$-stacking interactions. The induced scattering is thus mostly expected to be low and molecular dependent, in contrast to the electrochemical covalent functionalization.

In the remainder of this subsection we present a series of numerical studies on the influence of different disorder models on the transport properties of carbon nanotubes. Random uniform disorder is first approached, allowing analytic derivation of the mean free path scaling with the disorder strength. Then, semi-empirical methods are employed in assessing mean free paths or conductance functions in variously functionalized CNT from simple doping to defects and molecular chemiand physisorption. The final paragraph is dedicated to the problem of selective detection of molecules in CNT chem-FETs.

\subsubsection{Model Disorder: Basics of Elastic Mean-Free-Path Scaling}

For a better understanding of disorder effects, including inter-band scattering in the presence of short-range disorder [75], the evaluation of the elastic mean free path $\ell_{\mathrm{e}}$ is fundamental. For sufficiently weak disorder, a perturbative treatment can be performed within the Fermi golden rule (FGR). The FGR gives access to the elastic mean free path $\ell_{\mathrm{e}}=v_{\mathrm{F}} \tau$, with $v_{\mathrm{F}}$ the Fermi velocity and $\tau$ the mean free time. This was first derived by White and Todorov $[54,76]$ by reducing the band structure to a two-band approximation, as an effective model of the two degenerate bands at the charge neutrality point (CNP) for armchair nanotubes. By further considering an on-site Anderson-type disorder (see below), an analytical formula for $\ell_{\mathrm{e}}$ was derived; $\ell_{\mathrm{e}}$ was found to scale linearly with diameter for a fixed disorder strength $W$, whereas at a fixed diameter, the expected disorder scaling $\ell_{\mathrm{e}} \propto 1 / \mathrm{W}^{2}$ was shown. Considering the overall Hamiltonian as composed of the pristine CNT tight-binding Hamiltonian plus a small on-site perturbation $\hat{H}=\hat{H}_{0}+\hat{V}$, the application of the FGR yields 


$$
\frac{1}{2 \tau\left(E_{\mathrm{F}}\right)}=\frac{2 \pi}{\hbar}\left|\left\langle\psi+\left(k_{\mathrm{F}}\right)|V| \psi-\left(k_{\mathrm{F}}\right)\right\rangle\right|^{2} \rho\left(E_{\mathrm{F}}\right) \times N_{c} N_{\mathrm{R}}
$$

with $N_{\mathrm{c}}$ and $N_{\mathrm{R}}$ number of pair atoms along the circumference and the total number of rings taken in the unit cell used for diagonalization, respectively. The eigenstates of $\hat{\mathrm{H}}_{0}$ at the Fermi level can be written as $^{11)}$

$$
\left|\psi_{ \pm}\left(k_{\mathrm{F}}\right)\right\rangle=\frac{1}{\sqrt{N_{\mathrm{R}}}} \sum_{m=1}^{N_{\mathrm{R}}} \mathrm{e}^{\mathrm{i} m k_{\mathrm{F}}}\left|\alpha_{ \pm}(m)\right\rangle
$$

with

$$
\left|\alpha_{ \pm}(m)\right\rangle=\frac{1}{\sqrt{2 N}} \sum_{n=1}^{N_{c}} \mathrm{e}^{2 \pi i n / N_{c}}\left(\left|p_{z}^{A}(m n)\right\rangle \pm\left|p_{z}^{B}(m n)\right\rangle\right)
$$

The disorder considered here is an uncorrelated white noise (Andersontype) distribution given by

$$
\left\langle p_{z}^{\sigma}(m n)|\hat{V}| p_{z}^{\sigma^{\prime}}\left(m^{\prime} n^{\prime}\right)\right\rangle=\varepsilon_{\sigma}(m n) \delta_{\sigma \sigma^{\prime}} \delta_{m m^{\prime}} \delta_{n n^{\prime}}
$$

with $\sigma, \sigma^{\prime}\{A, B\}$, where $\varepsilon_{\sigma}(m n)$ are the on-site energies of electron at atoms $A$ and $B$ in position $(m, n)^{12)}$, having a random uniform distribution within the energy interval $[-W / 2, W / 2]$, and $\left|p_{z}^{\sigma}(m n)\right\rangle$ is a $\pi$-orbital centered on either $A$ or $B$ at ( $m$, $n)$. By substituting Equation (9) in Equation (8), and using Equation (10), a straightforward calculation gives

$$
\frac{1}{\tau\left(E_{\mathrm{F}}\right)}=\frac{\pi \rho\left(E_{\mathrm{F}}\right)}{\hbar}\left(\frac{1}{\sqrt{N_{\mathrm{c}} N_{\mathrm{R}}}} \sum_{N_{\mathrm{c}} N_{\mathrm{R}}} \varepsilon_{\mathrm{A}}^{2}+\frac{1}{\sqrt{N_{\mathrm{c}} N_{\mathrm{R}}}} \sum_{N_{\mathrm{c}} N_{\mathrm{R}}} \varepsilon_{B}^{2}\right)
$$

Hence, if the disorder is described by random fluctuations of on-site energies with uniform probability $1 / \mathrm{W}$ the mean free path can be finally analytically $[54$, 76] derived as

$$
\ell_{\mathrm{e}}=\frac{18 a_{c c} \gamma_{0}^{2}}{W^{2}} \sqrt{n^{2}+m^{2}+n m}
$$

with $a_{\mathrm{cc}}=1.42 \AA$ the $\mathrm{C}-\mathrm{C}$ bond length and $\gamma_{0}=2.7 \mathrm{~V}$ the typical TB hopping integral. For the armchair $m=n=5$ nanotube, with disorder $W=0.2 \gamma_{0}$, applying Equation $(12)^{13)}$, one finds $\ell_{\mathrm{e}} \approx 560 \mathrm{~nm}$, which is much larger than the circumfer-

11) That the same eigenstates have been used to obtain the STS image catalog in Figure 5.2 .

12) Here $(m, n)$ refers to integer translations along graphene lattice vectors $\mathbf{a}_{1,2}$. $A$ and $B$ are the labels of the two inequivalent atoms in the graphene unit cell.

13) In Equation (12), $(m, n)$ play again the role of the nanotube chiral index as opposed to Equation (10). 


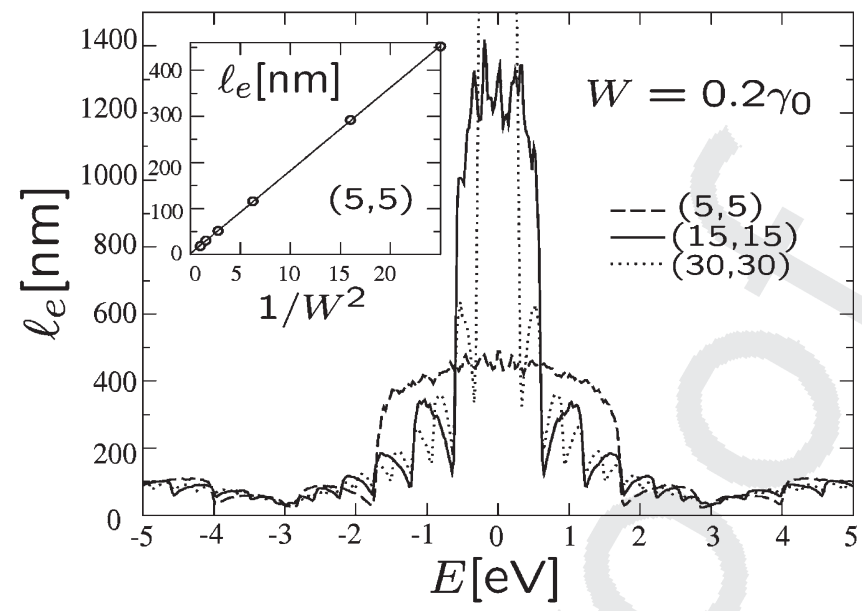

Figure 5.6 Energy-dependent mean free path as a function of diameter for different armchair carbon nanotubes. Inset: $1 / W^{2}$ scaling in agreement with Fermi golden rule. Adapted from Reference [55].

ence length. As shown in Figure 5.6, numerical studies [55] confirm the scaling law of the mean free path with the nanotube diameter close to the charge neutrality point. For semiconducting bands, the $1 / W^{2}$ is still satisfied, but mean free paths are seen to be much smaller and do not scale with diameter. With this information in mind, we move below to realistic disorders as created by dopants and adsorbed chemical species.

\subsubsection{Chemical Disorder or Doping: Conduction Mechanisms and Basic Length Scales}

The possibility to incorporate chemical impurities as substitutions of carbon atoms has been demonstrated experimentally [77] and offers novel possibilities to investigate coherent charge transport, and magneto-resistance phenomena in chemically modified carbon nanotubes. Substitutional doping by nitrogen or boron impurities has been a very intense research topic at the theoretical level during recent years [78, 79]. Initial work was focused on the effect of a single isolated defect on electronic and transport properties, while further studies have addressed the issue of mesoscopic transport in micrometer-long nanotubes with random distributions of impurities. These transport methods are mainly based on Kubo or Landauer-Büttiker frameworks, and mostly employed $a b$ initio calculations combined with semi-empirical $\pi-\pi^{*}$ Hamiltonians [80-82]. Such studies have allowed one to explore the fundamental elastic transport length scales [elastic mean free path $\left.\ell_{\mathrm{e}}(E)\right]$ and to investigate quantum interferences phenomena bringing the system from the weak to the strong localization regime.

To elaborate an effective tight-binding model able to describe the physics around the Fermi level, it is sufficient to describe properly the long-range scattering poten- 
(a)

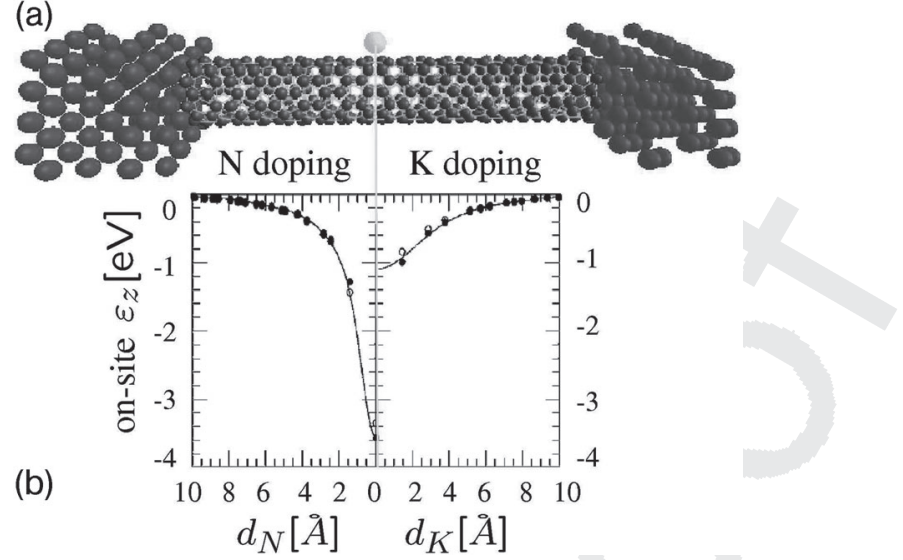

Figure 5.7 (a) Representation of a single adsorbed $\mathrm{K}$ atom on a CNT connected in between two metal contacts. (b) Longrange variations of the on-site and hopping parameters around $\mathrm{K}$ and $\mathrm{N}$ impurities derived from $a b$ initio calculations. Adapted from Reference [81].

tial due to the chemical impurities (assuming a low-density approximation, i.e. no possible interferences between tails of individual impurity potentials). The longrange variations of the on-site and hopping parameters around impurities can be directly derived from $a b$ initio calculations performed using atomic-like bases [81]. The tight-binding parameters obtained reproduce the position of the quasi-bound states in perfect agreement with the ab initio calculation [78]. For illustration, in Figure 5.7 the dependence of the $a b$ initio on-site Hamiltonian matrix elements associated with the $\mathrm{p}_{z}$ orbitals as a function of the distance to the impurity in a doped $(12 \times 12)$ graphene sheet is plotted [81]. The potential well created by $\mathrm{N}$ in substitution is clearly much deeper that the one associated with the partially screened $\mathrm{K}^{+}$ion. In particular, the ability of adsorbed $\mathrm{K}^{+}$ions to trap electrons is significantly reduced as compared with $\mathrm{N}$ impurities.

In Figure 5.8, the Landauer conductance computed from the $a b$ initio method (a) and the tight-binding model for a single nitrogen doped $(10,10)$ armchair

9 nanotube are shown [(b), inset]. At selected energies (inset: arrows), the conductance scaling properties are shown for a fixed impurity density $n_{\text {doping }}=0.1 \%$ (main plot). The extraction of the elastic mean free path $\ell_{\mathrm{e}}$ is achieved by adding the contribution of the ballistic term to the diffusive one, i.e. $R=1 / G=R_{0} / N_{\perp}+R_{0} /$ $\left(N_{\perp} L / \ell_{\mathrm{e}}\right)$, where $R_{0}=h / 2 e^{2}$ is the resistance quantum and $N_{\perp}$ the number of available transverse modes at a given energy. When $\ell_{\mathrm{e}} / L>>1$, the statistical distribution of $T$ is found to be narrowed and centered around $N_{\perp}$, in agreement with a ballistic limit $G=G_{0} N_{\perp}$, with $G_{0}=1 / R_{0}$ the conductance quantum. The other asymptotic case is found when $\ell_{\mathrm{e}} / L<1$, where the distribution of $T$ becomes wider with a mean value downscaling with the tube length as $\bar{T}(E)=N_{\perp}(E) \ell_{\mathrm{e}}(E) / L$. The conductance downscaling at a given energy exhibits a crossover from a ballistic to a diffusive regime. 


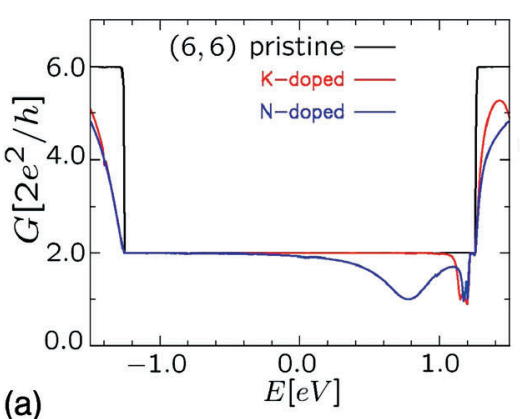

Figure 5.8 (a) Ab initio calculation of the conductance for a single nitrogen impurity in substitution of one carbon atom in the $(6,6)$ metallic nanotube [81]. (b) Length dependence of the Landauer conductance for the disordered $(10,10) \mathrm{N}$-doped nanotube at several energies (doping is fixed at $0.1 \%$ ).

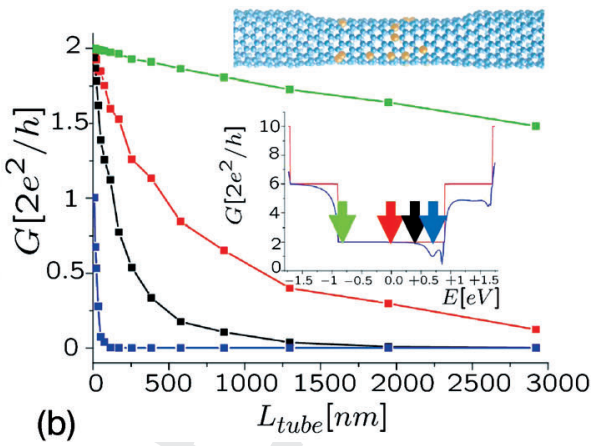

Inset: conductance versus energy for the perfect (dashed line) and single-impurity (solid line) cases for a single defect. Arrows show the considered energies for the scaling analysis (main frame). Adapted from Reference [82].

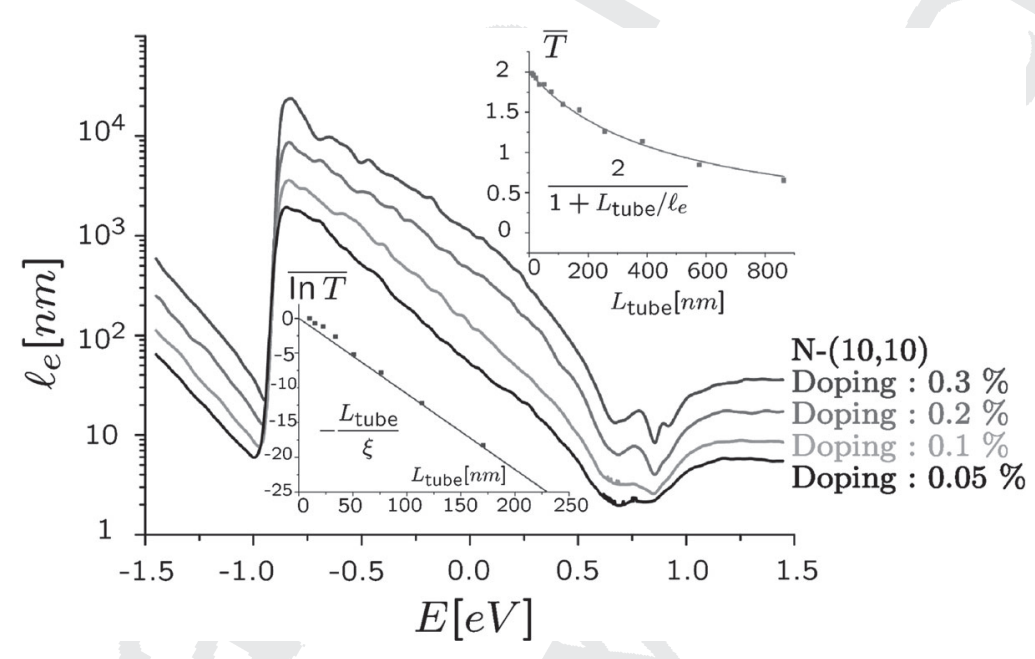

Figure $5.9 \ell_{\mathrm{e}}$ for several values of the doping density. Inset (top): $\bar{T}$ at CNP, for an average over 200 configurations. The linear fit (dashed) directly gives $\ell_{\mathrm{e}}$. Inset (bottom): $\overline{\ln T}$ at $E=$ $0.69 \mathrm{eV}$, with linear fit (dashed) giving access to $\tilde{\zeta}$ Both insets correspond to $n_{\text {doping }}=0.1 \%$. Adapted from Reference [82].

Figure 5.9 shows the full energy dependence of the elastic mean free path $\left(\ell_{\mathrm{e}}\right)$, evaluated from the Kubo formula, for several values of the doping density. It is readily shown that over the whole spectral window, $\ell_{\mathrm{e}} \propto 1 / n_{\text {doping, }}$, which is expected from the FGR. At the charge neutrality point, the scaling of $\ell_{\mathrm{e}}$ shows a linear increase with the radius of the tube $d_{\mathrm{t}} / 2$ as found in a simpler Anderson model of disorder (not shown here). The comparison between $\ell_{\mathrm{e}}$ extracted from both the 
Kubo and the Landauer formalisms are in very good agreement over the full spectrum at a quantitative level [82]. For instance, the top inset in Figure 5.9b shows the averaged transmission and the fitting curve $2 /\left[1+L / \ell_{\mathrm{e}}(E)\right]$, that yields $\ell_{\mathrm{e}}(E=$ $0.00)=495.5 \pm 17.4 \mathrm{~nm}$, in excellent agreement with the Kubo calculation that gives $\sim 460 \mathrm{~nm}$. For an energy at the frontier of the first sub-band below the CNP,

10 i.e. $E=-0.78 \mathrm{eV}$ (Figure 1a), the conductance slowly decays with length, and the regime remains quasi-ballistic. This is consistent with the calculated mean free path $\ell_{\mathrm{e}}(E=-0.78)=8371.6 \pm 69.4 \mathrm{~nm}$, which is much larger than the maximum length $(L=3000 \mathrm{~nm})$ of the tube in between contact probes.

For energies close to the nitrogen quasi-bound states (Figure 5.8), the impurityinduced backscattering becomes very strong, yielding a very small mean free path (see also Figure 5.9). On the "s wave" resonance, one actually finds that $\ell_{\mathrm{e}}(E$ $=0.69)=8.2 \pm 0.8 \mathrm{~nm}$. For lengths larger than the mean free path, the conductance

11 becomes exponentially reduced with length (Figure 5.7, curves c and d), defining a localized regime with $\tilde{\zeta}$ the length scale that quantifies the exponential decay of $\exp (\overline{\ln T})$ [83]. Accordingly, one obtains $\overline{\ln T}(E)=-L / \tilde{\zeta}(E)$ [82]. The next paragraph will go into more details concerning localization as expected in the presence of topological defects and covalent bonding of molecules on nanotubes.

\subsubsection{Defects, Covalent Functionalization and Anderson Localization}

The influence of defects is of fundamental relevance in the performance of electronic and sensing devices based on carbon nanotubes. Switching from a ballistic to either a weak or strong localization transport regime is possible above a certain density of defects. Moreover, as mentioned previously, defect sites augment the chemical reactivity of nanotube walls, rendering them sensitive to certain chemical species. Defects are most likely to be seen in nanotubes in different forms: topological defects, re-hybridization and incomplete bonding due to dislocations. Topological defects, distinguished by the presence of rings other than hexagons in the structure $^{14)}$ (e.g. the pentagon-heptagon pair or azulene structure), produces no net disclination but may slightly change the diameter and chirality of the tube, depending of its orientation relative to the tube axis. Knowing that a single sheet made of azulene as basic unit cell is metallic, as compared with the semi-metallic graphene, the introduction of pentagon-heptagon pair defects could close the band-gap of a nanotube and increase the metallic behavior [84].

Quantum transport theory [51] reveals that for a 1D conductor with defects, localization emerges whenever the "phase coherence length" $\ell_{\varphi}$ exceeds the localization length $\tilde{\zeta}$ For very long wires $(L \gg \tilde{\zeta})$, the electron transport is a diffusive process controlled by localization, with the electrons hopping between neighboring localized states. However, if $L$ is not too large $(L \approx 3-10 \tilde{\zeta})$ and the inelastic interaction is weak, the wire resistance is controlled by the phase-coherent electron propagation. This is the strong localization regime, in which the resistance increases exponentially with the length of the wire, $R \propto \exp (L / \tilde{\zeta})$ Depending on

14) Nanotube caps (closures) made of six pentagons according to Euler's theorem are not considered defects. 


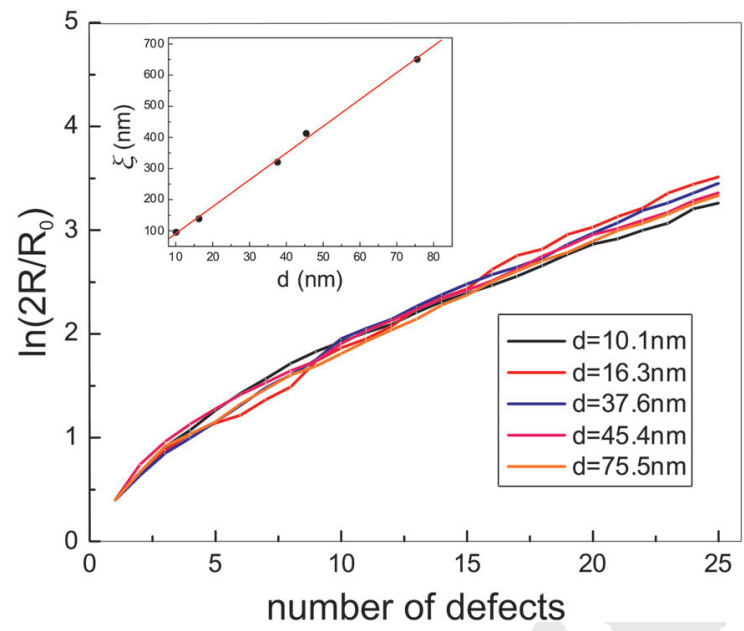

Figure 5.10 Calculated room temperature resistance for different average distances between di-vacancies $(d)$ as a function of number of defects. Inset: localization length $\tilde{\zeta}$, extracted from the fitting $R=R_{0} \times \exp (L / \tilde{\zeta})$ as a function of d. Adapted from Reference [86].

the defect density, in carbon nanotubes $\ell_{\varphi}$ can become longer than $\tilde{\zeta}$ driving the system into Anderson localization.

As an illustration, we show the extreme importance of defects (in particular divacancies) on the low-bias conducting properties of SWNTs irradiated with an $\mathrm{Ar}^{+}$ ion beam [85]. Only $0.03 \%$ of di-vacancies produce an increment of three orders of magnitude in the resistance of a 400-nm long CNT segment. Theoretical $a b$ initio calculations support this conclusion [86]. Indeed, for a $(10,10)$ carbon nanotube some of the findings are as follows: (i) the transition between the ballistic and the localization regimes installs for a small number of di-vacancies (about 35); (ii) for a higher number of defects the system shows localization, and the number of effective channels is reduced from two (ballistic) to one; (iii) at zero temperature, the nanotube conductance is strongly fluctuating, whereas the effect of finite $T$ is to wash out the fluctuations. The exponential scaling behavior, captured in Figure 5.10, is still preserved at room temperature. It is not clear whether electron correlation effects (e.g. Tomonaga-Luttinger liquid behavior) play a role and has to be resolved by future investigations.

We now focus on the covalent functionalization effects [87-90]. Starting from the occupied Bloch wavefunctions as obtained by ab initio DFT calculations on reference periodic systems, Marzari and coworkers $[87,88]$ extracted maximally localized Wannier functions (MLWF) to serve as basis sets for subsequent electronic structure and transport studies ${ }^{15)}$. The MLWF representation results in

15) Experience in parameterization of semi-empirical tight-bindinglike models reveals that realspace localized basis sets are transferable (portable) between systems of local atomic configuration similarity. 

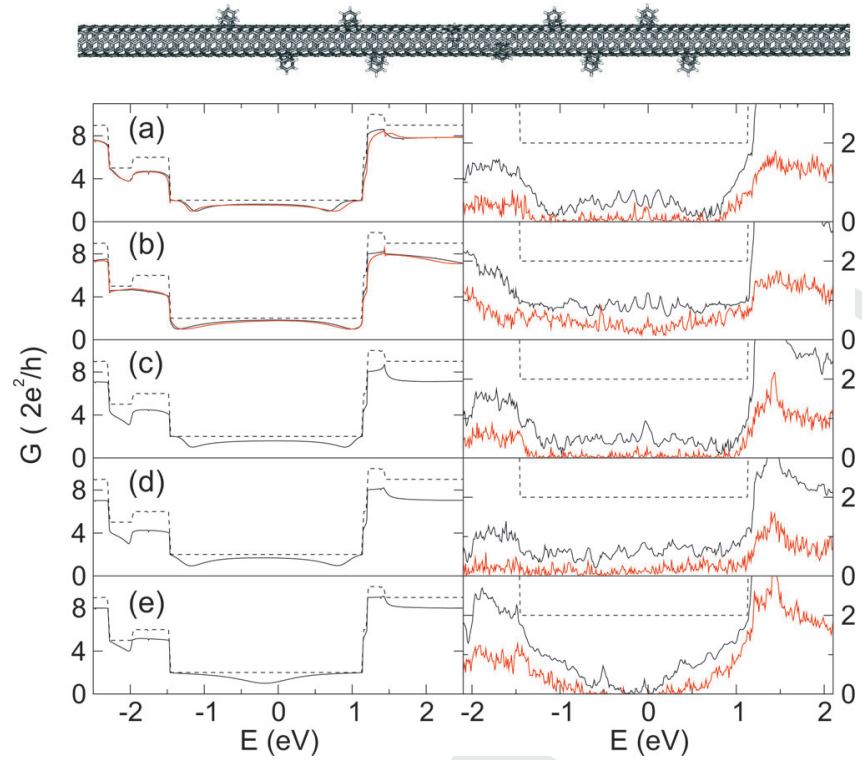

Figure 5.11 Top: Infinite metallic $(5,5)$ CNT functionalized by an array of phenyl pairs. Left: quantum conductance of an infinite $(5,5)$ CNT with one isolated pair of ligands in different positions $(a-d)$, or with a single ligand attached (e) (red line in a and b, hydrogen; solid line, model calculation;

dashed line, pristine CNT). Right: random distributions of pairs of ligands of of single ligands for the case of 10 defects per 1000 carbons (solid line) or 30/3000 (red line), averaged over five random configurations. Adapted from Reference [87].

sparse Hamiltonians which permit efficient implementations of the Landauer conductance calculation [see Equation (5)] and also the band dispersion everywhere in the Brillouin zone. The approach has been applied first to the study of covalent functionalization with electronegative or electropositive ligands such as nitrophenyls or aminophenyls in metallic SWNTs [87]. The band structure in the vicinity of the Fermi level is much less dependent on the chemical nature of the ligands (aminophenyl, nitrophenyl, phenyl and hydrogen), and seems rather affected by the $\mathrm{sp}^{3}$ functionalization pattern disrupting the conjugated $\mathrm{sp}^{2}$ network. Figure 5.11 displays several transport calculations. The ligands act as strong scatterers, reducing the conductance at the Fermi level by up to $42 \%$. As for the dispersion relations, the dominant factor is rather topological with almost identical results for hydrogen (see Figure 5.11a and b, red line] or the removal of $\mathrm{p}_{z}$ MLWFs from the CNT sidewall (solid line).

On the other hand, cycloadditions of carbenes and nitrenes offer a radically different avenue towards chemical control of the ballistic channels. Employing the same MLWF procedure, cycloadditions have been studied [88]. Figure 5.12, which compares the quantum conductance of the two stable open and closed configurations for the $(10,10)$ SWNT functionalized with $\mathrm{C}(\mathrm{CN})_{2}$, captures one of the central findings. The scattering induced by a single group is negligible (Figure 5.12a). 


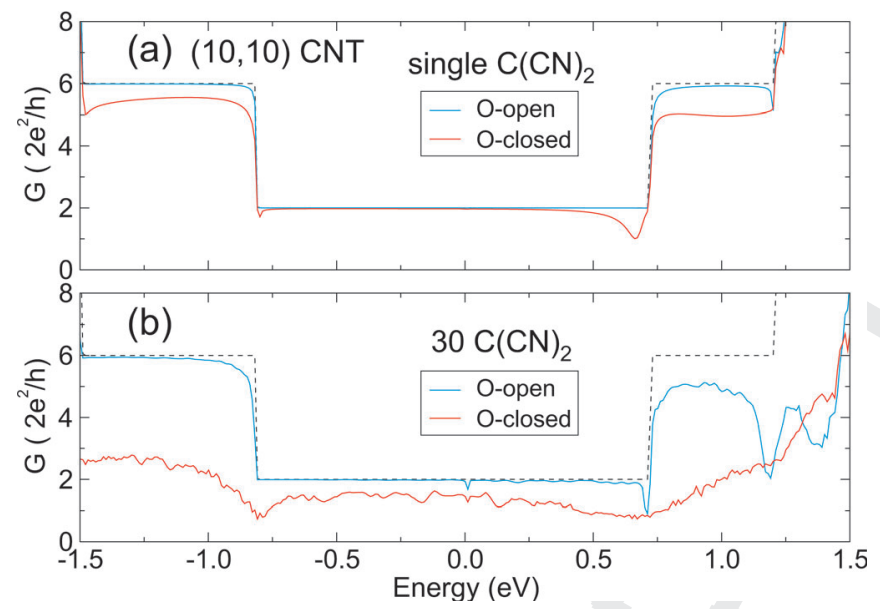

Figure 5.12 Quantum conductance for a $(10,10)$ CNT functionalized with $\mathrm{C}(\mathrm{CN})_{2}$, in the O-open and O-closed configurations (see [88] for details) [dashed line: pristine $(10,10) \mathrm{CNT}$. (a) Single group. (b) 30 functional groups randomly positioned on the central $32-\mathrm{nm}$ segment of an infinite tube (the conductance is averaged over 10 configurations). Adapted from Reference [88].

However, as the number of functional groups is increased, the difference between the two configuration increases considerably (Figure 5.12b). Therefore, one addition configuration (i.e. the O-open) allows for reversible cleaving of the sidewall bonds, and induces direct $\mathrm{sp}^{2}$ rehybridization, restoring the metallic conduction manifold. This observation allows a functionalization path preserving the electronic properties of CNTs around the Fermi level. A second strategy for minimally intrusive functionalization is physisorption, and constitutes the subject of the following section.

\subsubsection{Signatures of Physisorbed Molecules on Transport}

The effect of physisorption of small six-membered ring molecules $\mathrm{C}_{6} \mathrm{H}_{2 n}$ with $n=$ $3,4,6$, adsorbed on the CNTs has been investigated experimentally, through thermopower measurements. Sumanasekera et al. [17] reported on giant thermopower fluctuations due to the sorption of benzene molecules and attributed this effect to a strong $\pi-\pi$ overlap between orbitals. Such an approach addresses the intrinsic effect of molecular physisorption on quantum transport since the thermopower $S$ is related to the conductance modulations close to the Fermi level within the Mott equation. The validity of the Mott equation was further confirmed by Small et al. [91], even in the case of a Schottky-like injection regime.

Few theoretical studies have addressed the issue of molecular physisorption on the electronic properties of CNTs [92, 93]. To adjust the parameters of the semiempirical Hamiltonian operators, and since the interaction between the CNT and each molecule is geometry dependent, an $a b$ initio study of the adsorption needs 
(a)

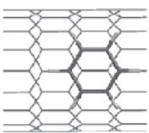

Bridge

(b)

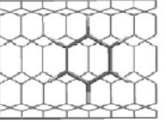

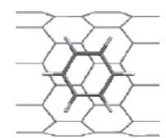

Bridge-bis

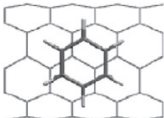

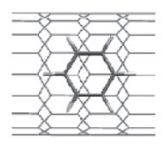

Stack

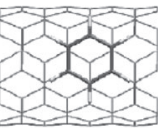

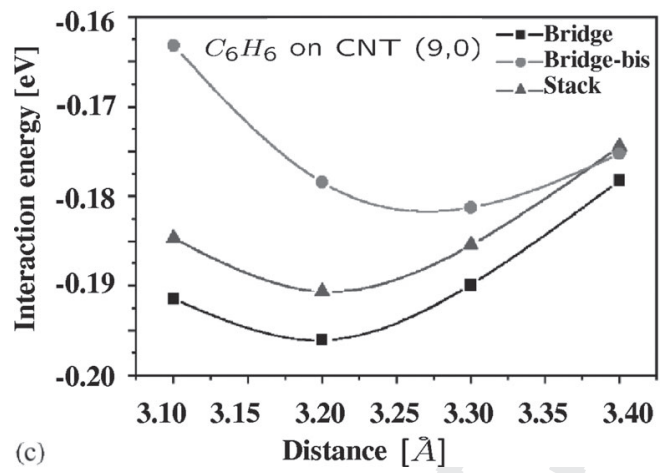

(c) Distance $[\AA]$

Figure 5.13 (a), (b) Different configurations considered for the adsorption of a benzene molecule on a $(9,0)$ zigzag CNT (a) and on a $(5,5)$ armchair. CNT (b). (c) Calculated interaction energy between a benzene molecule and a $(9,0)$ zigzag CNT. Adsorption curves corresponding to the "bridge" (squares), "bridge-bis" (circles), and "stack" (triangles) configurations. Adapted from Reference [92].

to be carried out prior to the TB analysis. Figure 5.13 shows the adsorption energy curves computed for a benzene molecule over a $(9,0)$ CNT in the configurations depicted in Figure 5.13a and b [92]. The two kinds of inequivalent $\mathrm{C}-\mathrm{C}$ bonds of the CNT lead to the two configurations named "bridge" and "bridge-bis" depending if the center of the benzene lies over the center of a bond parallel or not to the tube axis, respectively. In the "stack" configuration, the center of the molecule is just over an atom of the CNT. The most favorable structure is the "bridge" one, with the benzene molecule over a $\mathrm{C}-\mathrm{C}$ bond.

Electronic calculations have been performed on such a system using the DFT within its local density approximation (LDA) to predict the optimal geometry, and extract its electronic structure [94]. Calculations have been done using the aimpro code [95], with standard normconserving pseudo-potentials [96] and a $3 \times 3 \times 1 \mathrm{k}$ points sampling for the Brillouin zone integrations [92]. From the optimized geometries, the LDA band structures are plotted for the interactive system. The interaction acts as a mixing of the molecular single states with the underlying band structure, resulting in hybrid eigenstates, with low group velocity. However, due to the relatively large gaps of the isolated molecules $(5.217$ and $2.089 \mathrm{eV}$ for benzene and azulene, respectively), no strong modification is induced around the Fermi energy, and the linear band dispersion is maintained. 
The electronic properties of CNTs are described by the zone folding model, whereas the $\pi$-conjugated molecules are treated by the usual Hückel model [97]. The tight-binding Hamiltonian of the complete system, containing the interaction between CNT and $N_{\mathrm{M}}$ molecules, reads

$$
\hat{H}=\hat{H}_{C}+\sum_{m=1}^{N_{M}}\left[\hat{H}_{m}+\hat{V}_{C m}\right]
$$

where $\hat{H}_{C}$ is the CNT zone-folding Hamiltonian, and $\hat{H}_{m}$ is the Hückel Hamiltonian of the $m t h$ molecule. The remaining $\hat{V}_{C m}$ term corresponds to the coupling between the mth molecule and the CNT, and has the expression [94]

$$
\hat{V}_{C m}=\sum_{v \in C} \sum_{\mu \in m} \beta \cos \left(\varphi_{v \mu}\right) \exp \left(\frac{a_{g g}-r_{v \mu}}{\delta}\right)|v\rangle\langle\mu|
$$

The parameters of the coupling term were optimized to reproduce accurately the interaction between shells in multi-walled CNTs [33]. In Equation (14), $r_{v \mu}$ corresponds to the distance between sites and $\varphi_{v \mu}$ is the angle between the axes of two $|v\rangle$ and $|\mu\rangle \pi$-orbitals ${ }^{16}$. The parameters related to the interaction are $a_{\mathrm{gg}}=$ $3.34 \AA, \delta=0.45 \AA$ and $\beta=-0.36 \mathrm{eV}$. The tight-binding parameters (on-sites energies $\varepsilon$ and hopping integrals $\gamma$ ) are adjusted to reproduce the band structure of the graphene sheet and the energy levels of isolated benzene and azulene molecules, calculated within LDA. A standard procedure has been used to set the parameters for the graphene or the CNT, $\varepsilon_{C}=0 \mathrm{eV}$ and $\gamma_{C}=-2.56 \mathrm{eV}$, and also for the benzene molecule, $\varepsilon_{\mathrm{b}}=+0.411 \mathrm{eV}$ and $\gamma_{\mathrm{b}}=-2.61 \mathrm{eV}$. Owing to an inhomogeneous charge distribution along the azulene molecule, the TB model should be refined by adding an electrostatic correction. New on-site energies for the azulene molecule, proportional to the net charge on each carbon atom (calculated with LDA), have been calculated. With this approach, the TB value of the azulene HOMO-LUMO gap is $2.198 \mathrm{eV}$. Finally, the band structure computed with this modified TB model is compared with the previous ab initio results. As presented in Figure 5.14, this re-parameterized semi-empirical model gives an excellent description of the electronic states for both benzene and azulene adsorption cases.

The next step is the implementation of these parameters into an $O(N)$ TB calculation in order to study both the electronic structure [density of states (DoS)] and the transport (diffusion properties) in a CNT with a random coverage of noncovalently attached molecules. The computation of the quantum transport properties has been performed by computing the energy dependent diffusion coefficients of propagating electrons $D(E, t)$ \{see definition in Equation (4) [53]\}, whose time dependence determines the conduction mechanism and the elastic mean free path $\ell_{\mathrm{e}}$.

The DoS of a $(10,10)$ carbon nanotube with random coverage of adsorbed molecules are computed within this tight-binding framework. The density of grafted

16) For brevity in notation, $|v\rangle$ stand for $\left|p_{z}(m n)\right\rangle$ atomic orbitals as defined in Section 5.5.2.1. 


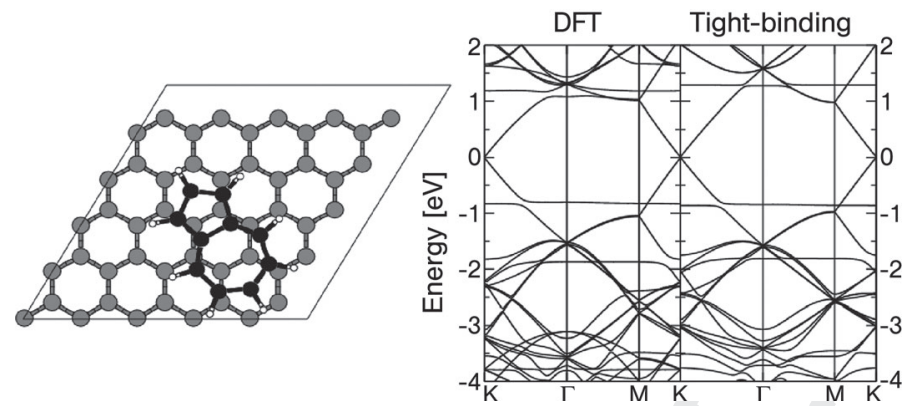

Figure 5.14 Right: The electronic structures of a graphene $5 \times 5$ supercell with one adsorbed $\pi$-conjugated molecule. Comparison between $a b$ initio and TB model. Left: picture of an azulene molecule physisorbed on the graphene sheet. Adapted from [94].
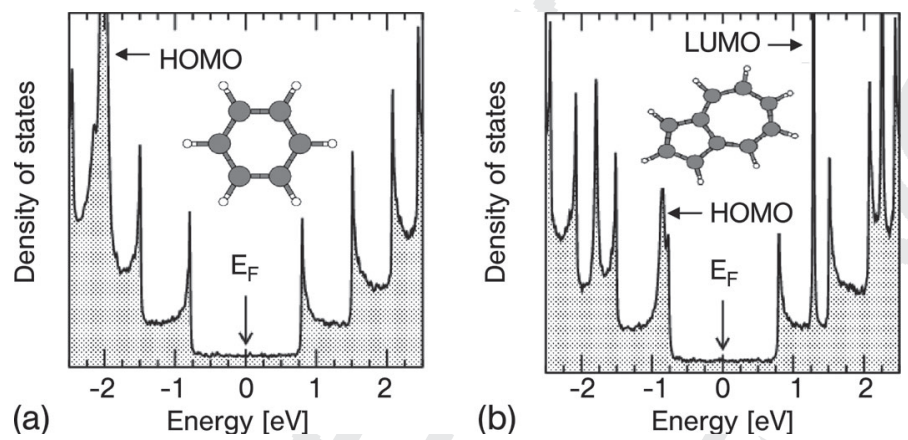

Figure 5.15 (a) DOS of the $(10,10)$ CNT with a benzene density coverage of $16.3 \%$. The HOMO molecular state is located by an arrow. (b) Same, but for an azulene density coverage of $11.5 \%$. HOMO and LUMO levels are identified by arrows. Adapted from Reference [94].

molecules is chosen to be $16.3 \%$ for benzene and $11.5 \%$ for azulene ${ }^{17)}$. Since the coupling intensity is weak, the DoS plotted in Figure 5.15 show the reminiscent discrete molecular levels, slightly enlarged by the mixing with the underlying continuum of $\pi-\pi^{*}$ bands. Peaks arising from molecular HOMO and LUMO levels are indicated. In the case of benzene adsorption, the DoS is weakly affected at charge neutrality point, and the computed diffusion coefficient does not depart from a linear scaling in time (ballistic regime). Elastic backscattering induced by benzene molecules is thus extremely weak at Fermi level (Figure 5.16a), which means that $\ell_{\mathrm{e}}$ is larger than several hundreds of microns (the considered limited tube lengths).

17) These densities correspond to the ratio of the mass of the adsorbed molecule and to the CNT mass. 

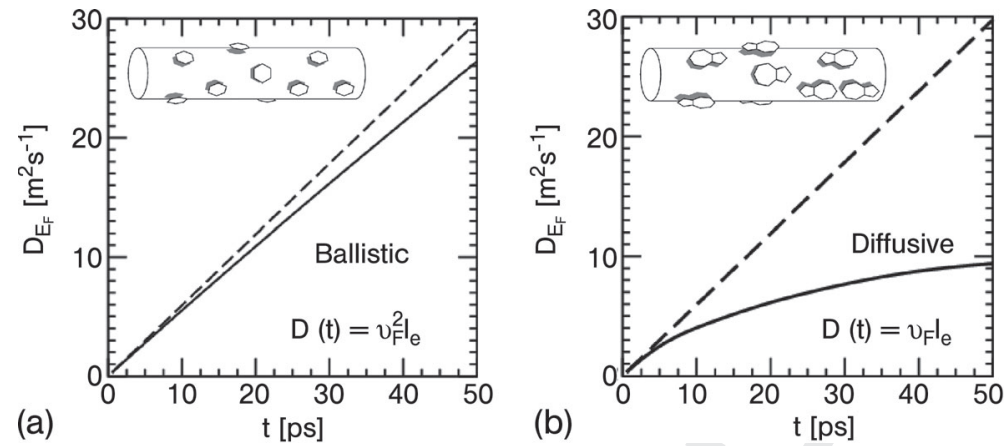

Figure 5.16 (a) Time-dependent diffusion coefficient (at the Fermi level) for the benzene/CNT system, showing quasiballistic behavior (solid line). Ballistic conduction for pristine CNT case is also reported (dashed line). (b) Time-dependent diffusion coefficient for the azulene/CNT structure, showing saturation at large times (diffusive regime). Adapted from Reference [94].

In contrast, in the case of azulene molecules, the HOMO level is located in the close vicinity of the last occupied Van Hove singularity of the CNT. Although the total DoS remains unchanged around the Fermi energy, the azulene adsorption impacts significantly on the intrinsic electronic conduction. In Figure 5.16b, the wave packet diffusivity at Fermi level is seen deviates more significantly from the ballistic regime. The saturation of $D(E, t)$ at long times allows one to extract the elastic mean free path $\ell_{\mathrm{e}}$, since $D(E, t \geq \tau) \approx v_{\mathrm{F}} \ell_{\mathrm{e}}$ (with $v_{\mathrm{F}}$ the Fermi velocity and $\tau$ the elastic mean free time).

Figure 5.17 gives the elastic mean free path scaling as a function of the azulene coverage density; $\ell_{\mathrm{e}}$ is roughly divided by a factor of 6 for a density increase of $\sim 30 \%$. One notes that in contrast to the substitutional chemical doping of carbon nanotubes, for which $\ell_{\mathrm{e}}$ is inversely proportional to the impurity density [80], $\ell_{\mathrm{e}}$ associated with the azulene physisorption shows a weaker dependence on the density of attached molecules. The origin of the obtained scaling law $\ell_{\mathrm{e}} \propto 1 \sqrt{\rho_{\text {mol }}}$ which deviates from the conventional Fermi golden rule remains unclear. These results have shown vanishing modulations of physisorption of aromatic molecules on the intrinsic conductance close to the Fermi level. The application of the Mott equation that gives the thermopower as $S \approx\left[k_{\mathrm{B}}^{2} T / e G\left(E_{\mathrm{F}}\right)\right]\left[\mathrm{d} G\left(E_{\mathrm{F}}\right) / \mathrm{d} E\right]$ has been demonstrated to be more general, however, including the effects of charge injection from external contacts [91]. In consequence, the strong modulations of thermopower in experiments [17] can hardly be attributed to physisorption effects provided that the Fermi level remains close to the undoped charge neutrality point. Possible modulations of the Schottky barrier at the metal/nanotube interface, or inter-tube coupling modulations, driven by benzene adsorption could be the possible origin of thermopower fluctuations.

On the other hand, the physisorption of azulene molecules demonstrates that such a weak interaction may produce substantial backscattering in the nanotube, 


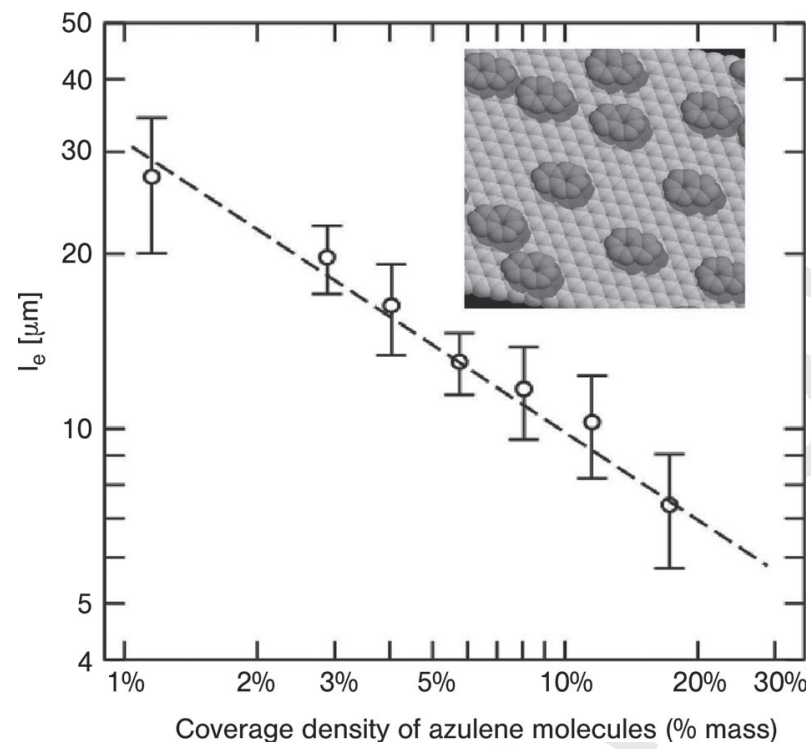

Figure 5.17 Mean-free-path of an azulene/CNT system versus the density of physisorbed molecules $\rho_{\text {mol. }}$. The dashed line shows $\ell_{\mathrm{e}} \propto 1 \sqrt{\rho_{\text {mol }}}$ Adapted from [94].

modifying its electronic mean free path and its conductance, which will downscale with the coverage density. Although the present study has been restricted to azulene molecules, other small gap $\pi$-conjugated hydrocarbon molecules such as fulvene should act in a similar way. Such a possibility of creating/removing a reversible elastic disorder by a simple adsorption/desorption of molecules covering the nanotube surface presents interesting perspectives for experimental studies and potential applications in nanotechnology.

\subsubsection{Sensor Specificity}

All examples treated so far, from the beginning of Section 5.5, show that carbon nanotubes are sensitive to many types of perturbations. In particular, several calculations showed that various chemical species could greatly influence the conductance of CNTs. With the huge advantage of sensitivity also comes the main challenge of CNT-based sensors, namely achieving selectivity, i.e. being able to distinguish the target in a sea of unwanted, perturbing signals. One possibility to improve selectivity is sensor functionalization such as with polymers [12] or singlestranded DNA [13]. In this subsection we investigate a second selective sensing strategy based on spectroscopic gate sweeps in CNT chemFET-like sensors giving access to the resonant molecular levels. A short first-principles discussion of zwitterion aromatic histidine (HIS), phenylalanine (PHE), tryptophan (TRP) and tyrosine (TYR) amino acids, bound through $\pi$-stacking on to a graphene sheet, is given below.

First, relaxation of the amino acids' atomic coordinates on graphene subject to weak $\pi-\pi$ interactions is achieved. Mean field theories such as Hartree-Fock (HF) 

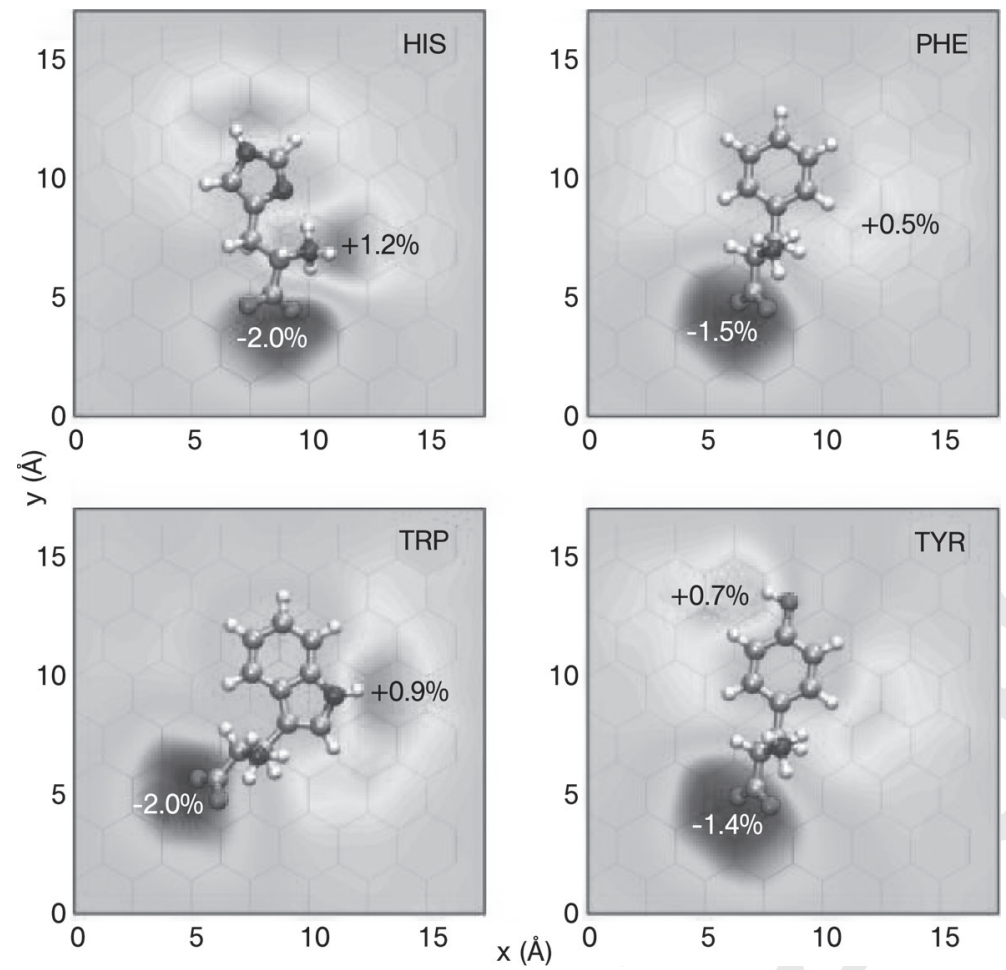

Figure 5.18 Mulliken charge transfer from/to $2 p_{z}$ orbitals of graphene. The figure also shows the underlying graphene lattice as an indication of the stacking configuration of the amino acids. Atomic charges are represented in real space by convolution with Gaussian kernels of $2.85 \AA$ effective cutoff centered at the atoms.

and DFT are known to have difficulties in predicting binding energies in the case of weak dispersion (London) forces. In fact, $\pi$-stacking is a benchmark for $a b$ initio electronic structure methods [98-101]. Improvements can be obtained in this case either through wavefunction correlation methods such as Møller-Plesset or coupled cluster [99, 101] or through DFT plus van derWaals corrections [102]. As mentioned in Section 5.4.1, all these methods involve a significant computational effort, further complicated by the basis set superposition error (BSSE) [103] issues, inherent in finite basis set calculations. Also, ab initio relaxation is costly for asymmetric molecules such as amino acids because of the large number of degrees of freedom. In this light, a classical force-field CHARMM as implemented in the code NAMD [104] along with MacKerell et al.'s [105] set of amino acid parameters is preferred. van derWaals parameters for graphitic carbon are borrowed from benzene. The geometries obtained after conjugate gradient minimization, are displayed in Figure 5.18. Except for tryptophan, which relaxed into a "stack" configuration because of the two aromatic rings, the other three amino acids relaxed into a "bridge" configuration. This together with a mean ring-graphene distance 


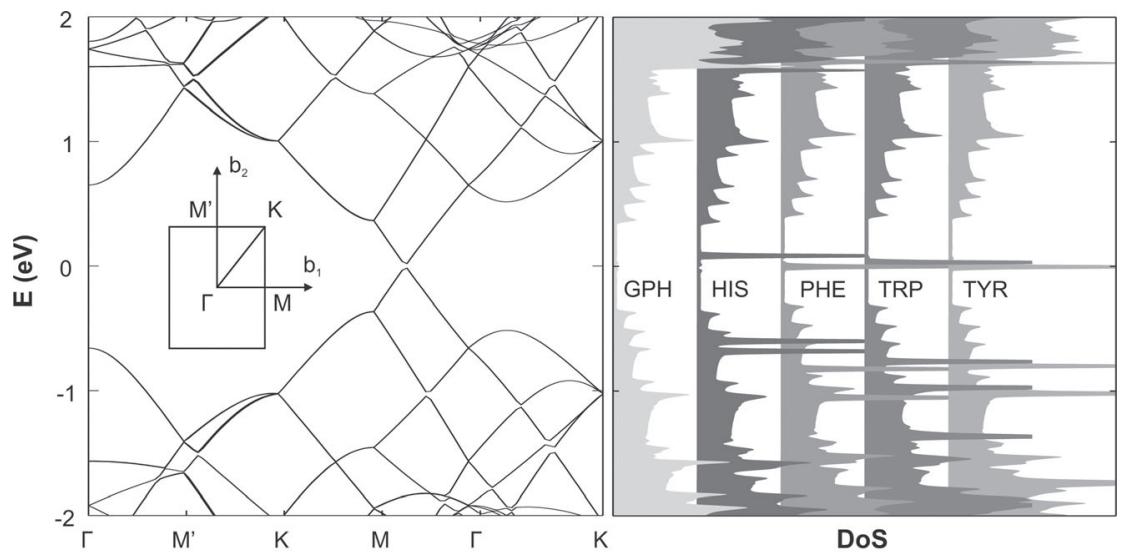

(a)

(b)

Figure 5.19 (a) DFT band structures of a graphene rectangular super-cell, along the symmetry lines defined in the first Brillouin zone sketched in the inset. (b) Total density of states for pristine graphene and the four adsystems emphasizing the amino aciddependent spectral peaks close to the Fermi level. van Hove singularities are apparent due to integration of DoS only along the symmetry lines.

of approximately $3.3 \AA$, confirms the validity of the molecular mechanics relaxation method.

For each ad-system consisting of one aromatic amino acid on to a graphene layer, $a b$ initio self-consistent calculations were performed with the DFT code SIESTA [106]. Since the systems are slabs (2D), an $8 \times 8 \times 1$ Monkhorst-Pack $k$ grid is chosen for sampling the rectangular first Brillouin zone with $32 k$ points. In Figure 5.18, Mulliken charges as computed with SIESTA are displayed. It is well known that Mulliken charges are strongly dependent on the basis set. Moreover, since relaxation at the ab initio level and counterpoise correction have been ignored, the charge analysis might further suffer from BSSE. Nevertheless, the charge maps presented in Figure 5.18 can be accepted qualitatively as they correlate well with the expected amino acid charges. The magnitude of the transferred charges ${ }^{18)}$ places the system in the physisorption regime. The charge is mostly transferred from/to the $2 \mathrm{p}_{z}$ orbitals, whereas for the other orbitals the transfer is typically five times lower. A Fermi level shift $(\sim 150 \mathrm{meV}$ up-shift or electron doping) together with the charge transfer from the $2 \mathrm{p}_{z}$ orbitals and the localized states close to $E_{\mathrm{F}}$ are mechanisms that can result in important conductance changes. Therefore, we suggest that carbon nanotubes might be suitable for aromatic amino acid detection.

Total density of states (DoS) are displayed in Figure 5.19. The presence of localized amino acid-dependent states close to the Fermi level is evident. These states

18) The peak charge transferred is about $2 \%$ electrons distributed over 3-4 carbon atoms (see Figure 5.18). 
are found to populate the $\alpha$-carboxyl group orbitals of the amino acids and not the aromatic ring orbitals. The latter are populated significantly only by states found $3 \mathrm{eV}$ away from the Fermi level and cannot, at least in theory, influence the intrinsic conductance of a carbon nanotube (see also Section 5.5.2.4 for a discussion of $\mathrm{C}_{6} \mathrm{H}_{6}$ physisorption on graphene). Hence it can be concluded that although responsible for the binding mechanism, it is not the aromatic rings of the amino acids that could change a nanotube's conductance but rather the carboxyl or amino ions. However, the most important feature is that the spectral position of the localized states is dependent on the amino acid type. These individualized signatures could prove extremely useful in the context of amino acid identification by CNT chemFETs. In sweeping the gate voltage, whenever $E_{\mathrm{F}}$ would cross these localized state resonant energies, a peak in the low bias conductance could be detected. This spectroscopic information can be used to infer the analyte, alleviating one of the known weaknesses of carbon nanotube-based sensors.

\subsection{3}

\section{An Electromechanical Displacement Transducer}

In this final case study, we present some modeling efforts aimed at validating the operation of a CNT-based electromechanical transducer. The analyses performed are different from the previous ones, in that device characteristics are targeted instead of intrinsic nanotube properties. This transducer, consisting of a CNT cross junction, has the principal function of measuring nanometric displacements. Relying on this basic function, both weak forces $(\mathrm{pN})$ and small mass $\left(\mathrm{kDa}^{19)}\right)$ detection can be implemented, based on measurement principles detailed below.

The transducer studied is sketched in Figure 5.20 and consists of two perpendicular carbon nanotubes in a cross configuration. One nanotube is a doubly clamped linear bearing and the second is a singly clamped cantilever beam. The bearing is placed underneath the cantilever to prevent its collapse and stiction to the substrate by van der Waals attraction. The three metallic contacts, to which the tubes are clamped, serve also to bias the device electrically. For this study, a simple DC biasing scheme has been adopted, with the two terminals of the bearing set to $+V$ (where $V$ is some potential) and the single terminal of the cantilever set to $-V$. In the steady state, when no external force is applied on the cantilever, the currents flowing through the two branches of the bearing should be approximately equal $^{20}$. Whenever the cantilever bends, the length and consequently the current ratio of the two branches change. It is this branch current imbalance which is "measured" in order to obtain the deformation of the cantilever. A note is, however, important here. As seen in Section 5.5.2, for a ballistic tube the conductance does not scale with length so regardless of the cantilever's deflection no substantial

19) $1 \mathrm{kDa}=1.65 \times 10^{-21} \mathrm{~g}$.

20) In practice there will always be an offset owing to unequal branch lengths or differences in the doping or structural imperfections of the branches. 

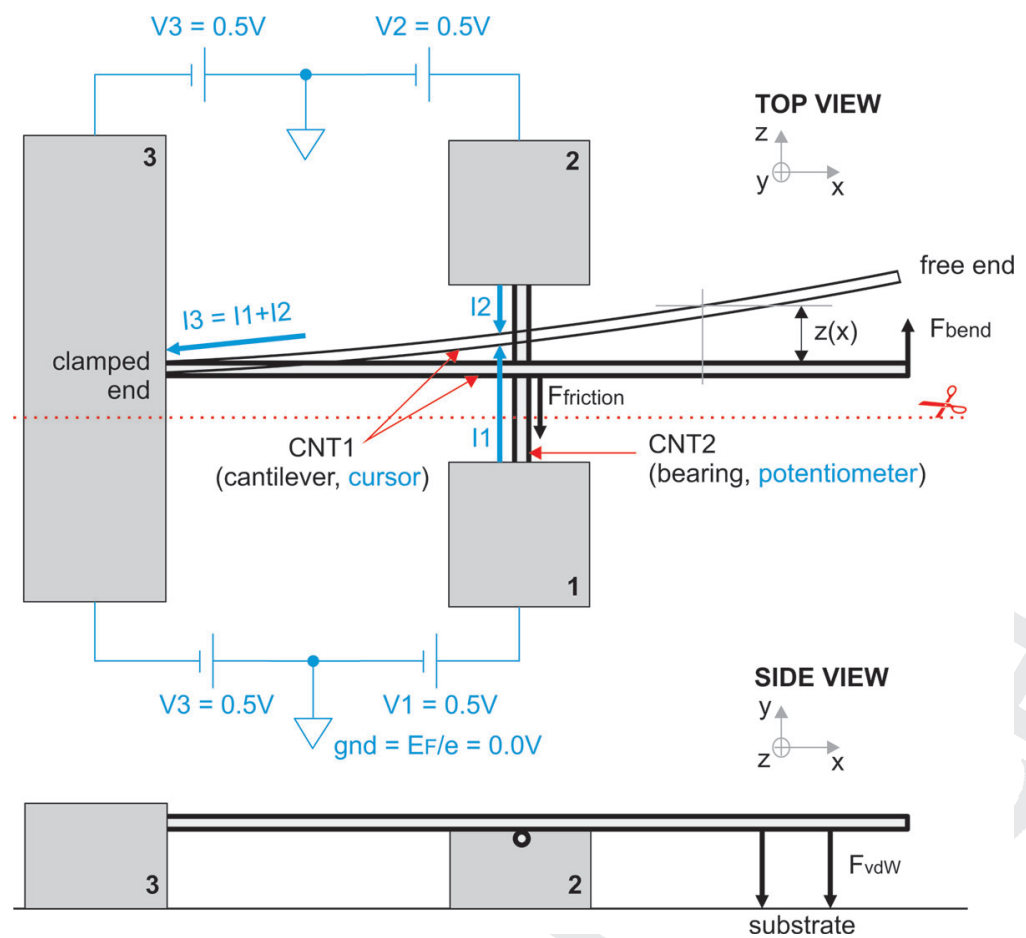

Figure 5.20 Schematic representation of the operation principle of a carbon nanotube-based displacement transducer. Top view: the bearing is biased to $+V$ and the cantilever to $-V$. In blue lines the electrical part is plotted, whereas mechanics are plotted in black lines. Bottom view: cross-sectional side view along the dashed red line. Adapted from Reference [107].

branch current difference should be recorded. Therefore, some scattering should be present in the bearing to force length scaling, achievable in practice by doping or inducing structural defects (e.g. by ion bombardment; see Section 5.5.2.3).

Measuring forces with this transducer is straightforward. The cantilever's deflection $\Delta z$ is transformed into a differential current $\Delta I=I_{1}-I_{2}$, which is captured by readout electronics. Both the magnitude and the sign of the external force $F$ can be obtained by multiplying the spring constant of the system with the previously determined deflection $\Delta z$, i.e. $F=k \Delta z=(3 Y I) / L^{3} \Delta z$, where $Y$ is Young's modulus, $I$ the moment of inertia and $L$ the length of the cantilever tube. Regarding the second application, i.e. minute mass weighting or molecular detection, the transduction of a molecular binding event into an electrical signal is based on the oscillating frequency difference between the fundamental mode of the free cantilever versus the cantilever with a body attached. Measuring this frequency shift yields information about the mass of the attached body, which could be a macro- 
molecule, virus or any other small particle. This device configuration is named hereafter nanobalance. In the following, we concentrate on the modeling approach taken in order to obtain the different device characteristics. The modeling tasks are split in two, one set being related to the mechanical response and the other to charge transport.

\subsubsection{Mechanical Transducer Behavior}

Section 5.4.3 reviews some of the available choices for modeling the mechanical properties of SWNTs. As an illustration, here we discuss the model choice made, in view of the particularities of the system studied. The continuum Euler-Bernoulli beam theory [109] has been validated in moderate strain regimes for nanotube-based AFM tips [110, 111], nanotweezers [112], electromechanical resonators [20] and nanoswitches [113]. However, atomic-scale fluctuations of the van derWaals potential coupled with the position-dependent shape of the cross junction yield pseudo-random friction forces. An atomistic description of the system in the framework of molecular dynamics (MD) is therefore imposed, which results in a new decision, namely choosing the appropriate force field (FF). Carbon nanotubes were studied using various FFs ranging from ab initio [114], tight-binding [115], Tersoff-Brenner [116] and even classical force fields such as CHARMM [117] (see Section 5.4.3). Nevertheless, only the last two FFs can deal with more than $10^{6}$ atoms for a few nanoseconds of simulations. Compared with the Brenner potential, successful in describing carbon-carbon interactions, the CHARMM FF is additionally parameterized for a large spectrum of organic molecules, notably amino acids and phospholipids [105]. This advantage is exploited in the simulation the transducer in contact with a protein (streptavidin) later in this section. CHARMM is also considerably faster.

Since force fields in CHARMM's class have been used mostly for modeling CNT hydrophobic-hydrophilic effects [117], a new set of carbon parameters are derived that reproduce mechanical properties within a reasonable strain. The parametrization procedure, detailed elsewhere [107], relies on DFT $a b$ initio calculations as performed with SIESTA [106]. Briefly, from the relaxed atomic coordinates in the unit cell of a $(5,5)$ armchair CNT, the equilibrium bond length, bond angle and second neighbor distance (or Urey-Bradley term), i.e. $r_{0}, \theta_{0}$ and $r_{0 \mathrm{UB}}$, respectively, are obtained. Subsequently, the unit cell has been subjected to tensile strains in the $[-10,10] \%$ range with a $1 \%$ step, and the total energy was calculated with SIESTA after relaxation at each strain. The rest of the CHARMM force-field parameters, namely the spring constants $k, k_{\theta}, k_{\mathrm{UB}}$ and the Lennard-Jones well-depth $\varepsilon_{\mathrm{vdw}}$, were fitted by matching the total energy vs. strain curve as calculated with CHARMM against the $a b$ initio one. Figure 5.21 shows the good agreement between the two curves with the final, optimized CHARMM parameters [107]. The Young's modulus extracted from the DFT curve ${ }^{21)}$ corresponds to $1.04 \mathrm{TPa}$, which is a realistic value for this type of nanotube.

21) The Young's modulus is defined as the second derivative of total energy with respect to tensile strain, divided by the volume of the unit cell, $Y=(1 / V) \mathrm{d} E / \mathrm{d} \varepsilon$. 


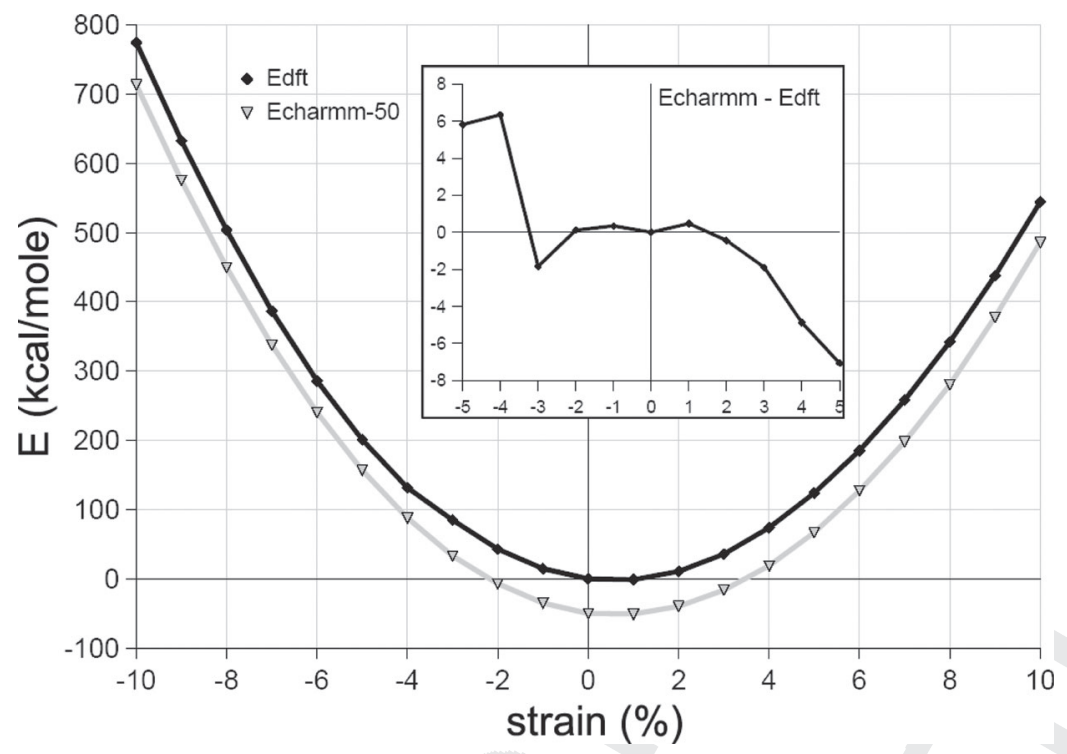

Figure 5.21 Energy-strain characteristics as obtained with DFT and the molecular dynamics CHARMM force field. The inset details the error around origin. Curves have been shifted vertically by $50 \mathrm{kcal} / \mathrm{mol}^{-1}$ for better visualization. Adapted from Reference [107].

With the parameterized CHARMM force field, several MD simulations have been performed to assess the dynamic behavior of the transducer. In the first MD simulation, a cantilever measuring $36 \mathrm{~nm}$ was pushed with a constant force of $10 \mathrm{pN}$ evenly distributed between its 10 terminal atoms while clamping the other end of the tube. Turning on the force at the initial simulation step is equivalent to applying a unit-step stimulus, that excites simultaneously all the modes of the cantilever. For instance, the continuous component of the spectrum gives the final displacement as would be obtained in the presence of dissipation. Subsequently, three different positions of the $(5,5)$ bearing tube, measuring $20 \mathrm{~nm}$, were chosen to study the influence of the friction: at one-third half and two-thirds of the distance from either edge of the cantilever. The simulation step of the molecular dynamics was set to $1 \mathrm{fs}$ and the total simulation time was limited to $0.5 \mathrm{~ns}$ (corresponding to $2 \mathrm{GHz}$ ). This time interval proved to be sufficient for capturing at least one period of the cantilever's fundamental mode (see Figure 5.22) in all four situations, but not long enough to allow the extraction of the superior modes via Fourier analysis, or the quality factor. The fundamental's mode frequency of the free cantilever agrees well with the classical Euler cantilevered beam value of $2.17 \mathrm{GHz}$. As opposed to the free cantilever case, the bearing adds friction. The mechanical work done to move the cantilever against friction, transforms to heat, as can be observed in Figure 5.22a. Although the magnitude of the friction forces is difficult to obtain from these data, it is obvious that friction is well below $10 \mathrm{pN}$, 

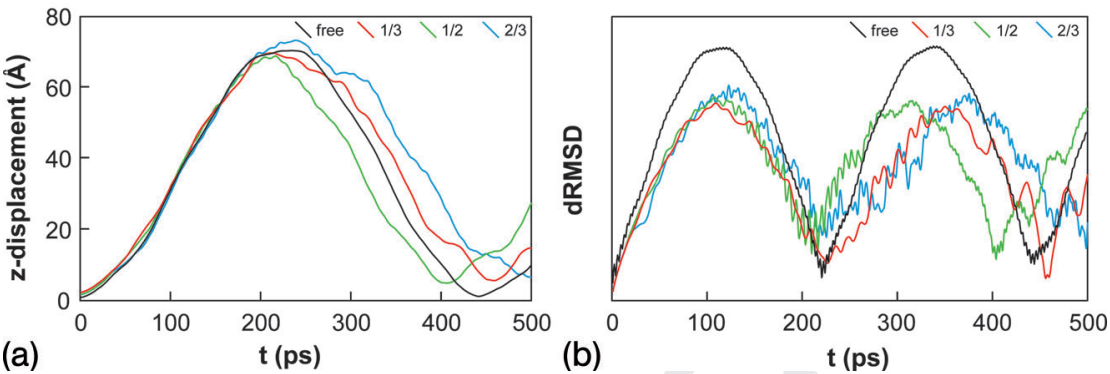

Figure 5.22 Different curves relevant to the mechanical response of the strain transducer. (a) Cantilever's deflection along the $z$-axis (the coordinate of one atom found on the tip). (b) Time derivative of the root mean square displacement (RMSD) of all atoms of the cantilever. Adapted from Reference [107].
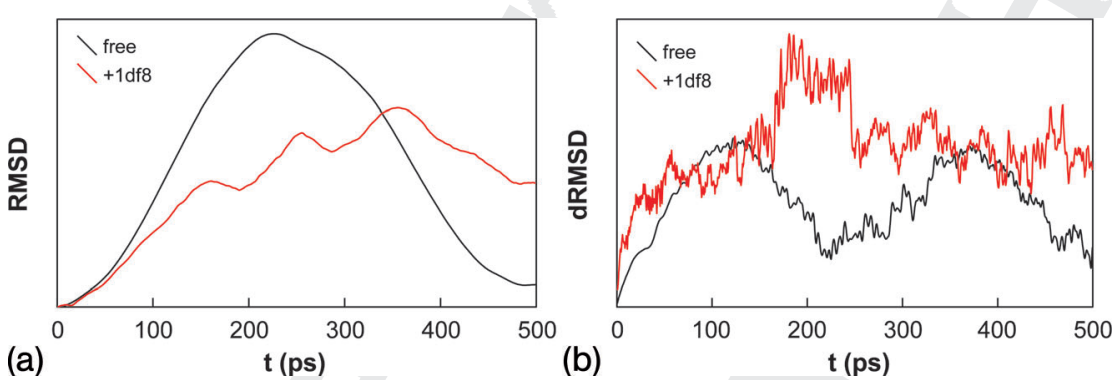

Figure 5.23 Normalized root mean square displacement (a) and its time derivative (b) for the free transducer and a single streptavidin molecule (1df8) attached at the tip of the cantilever. Adapted from Reference [108].

and does not influence the dynamics of the cantilever too much. Therefore, the presence of the bearing can be ignored from a mechanical point of view, which simplifies things considerably, and allows a simple Euler beam or other continuum theory to be used for the transducer mechanics.

We now turn our attention to the nanobalance configuration of the transducer. To demonstrate that this device is in principle capable of detecting minute masses, resonant frequency shifts are evidenced whenever an external body (typically a molecule) binds to the cantilever tube. The system under study is the biotin-streptavidin complex. Biotin can be modified to accommodate non-covalent binding on the surface of the nanotube. As before, MD is used for simulation to probe the frequency response of the system, with and without attached streptavidin. The bearing was placed at one-third of the distance from the cantilever's tip, whereas one streptavidin molecule was attached to the tip. Figure 5.23 shows that the cantilever's deflection in the molecule-attached case is retarded with respect to the free cantilever. This proves that the transducer is sensitive enough to detect a 
single molecule of streptavidin, which weighs only a few $\mathrm{kDa}$. The relative frequency drop $\Delta f / f_{0}$ estimated from Figure 5.23 is approximately $35 \%$, which is rather important. Already seen in the RMSD (a), but highlighted in the dRMSD (b), is the abundance of additional modes introduced by the protein appearing noise-like, reducing the quality factor of the system.

\subsubsection{Transport Strain-Current Characteristic}

The molecular mechanics simulations described in the previous section focused on the operation of the transducer from the mechanical point of view. Here we switch to the investigation of the transport characteristics of the transducer, which tries to demonstrate that a deflection of the cantilever transforms into a branch current difference as explained in Figure 5.20.

The physics of CNT cross junctions is rich. The tunneling current through the junction depends strongly on the inter-tube distance, which fluctuates considerably at room temperature. Since the tunneling current is relatively weak, electrons tend to accumulate on the cantilever where they dwell for a while before disappearing into the bearing contacts. Charge shuttling and phonon-assisted tunneling may therefore be relevant, together with other potential many-body effects (e.g. correlated electron dynamics). Modeling transport through CNT cross junctions is rendered difficult - apart the complex physics - by the large number of atoms involved (a few thousand in this case). Achieving charge self-consistency is therefore too difficult, imposing a simplified tight-binding description. Due to the short lengths of the nanotube segments ${ }^{22}$, it is safe to assume that the intra-tube transport is ballistic. At the junction, phonon-assisted tunneling is neglected. Finite temperature effects are, however, taken into account by averaging over the ensemble of nuclear positions when computing branch currents from the transmission spectra.

The scheme used in transport calculations is similar to Reference [58], with some extensions, such as including multiple leads and taking into account the non-covalent tunnel junction. The Hamiltonian model is basically a $\pi$-only nearestneighbor tight-binding model for intra-tube interactions plus a Slater-Koster-type cosine factor to account for the anisotropic inter-tube coupling (see also the model in Section 5.5.2.1):

$$
\begin{aligned}
\hat{H}= & \sum_{v \in \mathrm{T}_{1,2}} \varepsilon_{0 v}|v\rangle\left\langle v\left|+\sum_{v \in \mathrm{T}_{2}} \delta \varepsilon_{0 v}\right| v\right\rangle\left\langle v\left|+\sum_{v, \mu \in \mathrm{T}_{1,2}} \gamma_{0} \exp \left(\frac{a_{\mathrm{CC}}-r_{v \mu}}{d}\right)\right| v\right\rangle\langle\mu| \\
& +\sum_{v \in \mathrm{T}_{1}} \sum_{\mu \in \mathrm{T}_{2}} \beta \cos \left(\varphi_{v \mu}\right) \exp \left(\frac{a_{g g}-r_{v \mu}}{\delta}\right)|v\rangle\langle\mu|
\end{aligned}
$$

The first term is a sum over the on-site energies $\varepsilon_{0 v}=\varepsilon_{0}-\mid e / V(v)$ of all atoms in the tubes $\mathrm{T}_{1,2}$. Electrostatics is included via $|e| V(v)$, the field created by the leads.

22) The longest nanotube segment measures $36 \mathrm{~nm}$. 


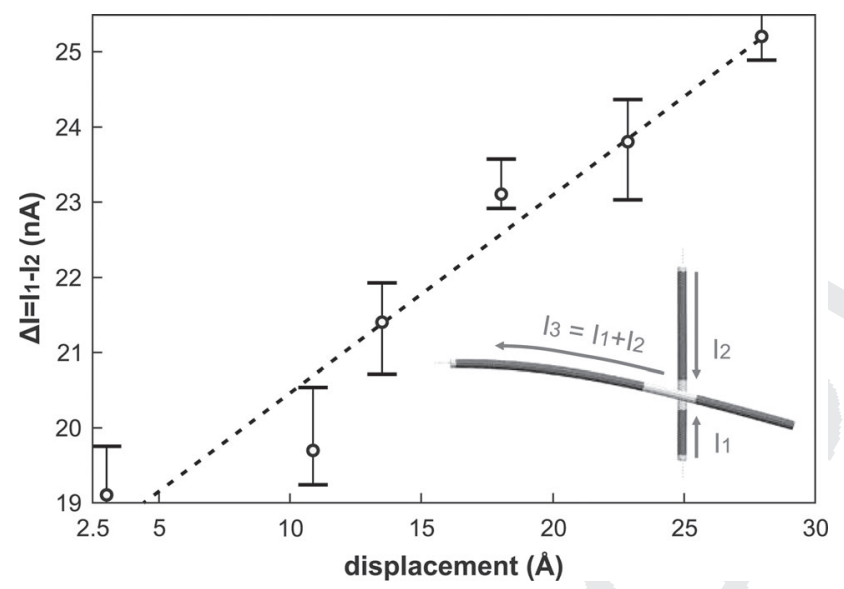

Figure 5.24 Overall current-deflection characteristic of the sensor showing a monotonic trend. Each point is obtained by averaging over thermally randomized configurations (error bars displayed). Inset: atomistic structure of the sensor and real-space partition used for quantum transport calculations. Adapted from Reference [107].

Both nanotubes are assumed to be in equilibrium with their metallic contacts, and therefore $V(v)$ is set constant along each tube. The second term contains a uniform disorder of on-site atom energies $\delta \varepsilon_{0 v}$, sampled from the interval $[-1,1] \mathrm{eV}$, and applies only to the bearing atoms $\left(\mathrm{T}_{2}\right)$. This disorder is added to induce conductance length scaling in the bearing by localization. Strain in the cantilever is taken into account via the exponential modulation with a $d=2 \AA$ decay length, where $r_{v \mu}$ represents the distance between atoms $\mu$ and $v$ and $a_{C C}$ is the equilibrium $\mathrm{C}-\mathrm{C}$ bond length ( $1.42 \AA)$. The last sum describes inter-tube coupling, and includes the effect of non-parallel $\pi$-orbital axes via the cosine term, and finite interaction range via the second exponential factor with $\delta$ decay length ${ }^{23)}$.

Contact currents have been calculated from Green's functions with the multiterminal Landauer-Büttiker equation. The methodology utilized is explained elsewhere [107]. To avoid a direct inversion of the system's Hamiltonian, nanotube segments outside the junction region have been cut into thin rings, resulting in a block-chain matrix, for which fast, order- $N$ elimination techniques work well [57]. The only matrix inversion remaining is the calculation of the junction-region Green's function, which is small enough not to penalize the simulation time. In order to take into account the atomic fluctuations at room temperature, 25 closely located junction configurations were sampled from the molecular mechanics

23) The values of the different parameters are the same as, and the Hamiltonian model is almost identical with, the model described by Equation (14). 
simulation. The length of the distribution interval was of $\sim 2 \AA$, consistent with thermal displacement fluctuation amplitudes as given by the equipartition theorem applied to the modes of a classical cantilever. The currents computed for each configuration were subsequently averaged and yielded Figure 5.24. An overall monotonic increase is clearly visible in Figure 5.24, which plots the current difference between the two branches of the bearing at different cantilever deflections. Although this characteristic can be used in validating the operation principle of the transducer, the model described thus far should not be interpreted quantitatively but rather qualitatively.

\section{6}

\section{Conclusions and Perspectives}

There is a great variety of applications for which nanotubes represent a disruptive potential, ranging from energy storage, composites, nanoelectronics and other solid-state devices, to sensors and actuators. This chapter has attempted to highlight the importance of bringing together theoretical modeling and experimental studies in the highly dynamic and complex field of CNTbased sensors. In the bio and medical fields, where target drug delivery and monitoring of specific enzymatic reactions are very important, the advantages of CNTs over traditional technologies could lead to genuine scientific and technological breakthroughs.

Under a brief examination, nanotube transducers have revealed a wide palette of sensing mechanisms, exploiting the exceptional structural, electronic and mechanical properties of CNTs. To be able to cope with the different timescales, feature sizes and phenomena present in nanotube systems, theory has often to rely on hybrid or multi-scale modeling. By producing reliable multiscale models, with real predictive value for device design, theory could assist decisively the technological efforts to overcome the remaining challenges, and promote carbon nanotubes as the material of the future in industrial applications.

\section{7}

\section{Acknowledgments}

We are grateful for fruitful discussions with V. Crespi, M. Cohen, S. G. Louie, P. M. Ajayan, R. Avriller, X. Blase, J. C. Charlier, S. Latil and F. Triozon and acknowledge funding from the European Community through NoE Nanoquanta (NMP4CT-2004-500198), SANES (NMP4-CT-2006-017310), DNA-NANODEVICES (IST-2006-029192) and NANO-ERA Chemistry projects, UPV/EHU (SGIker Arina) and the Basque Governement. The work published here by C.R. was done during his thesis studies at Tima Laboratory, Grenoble, France. A.R. gratefully acknowledges the computer resources, technical expertise and assistance provided by the Barcelona Supercomputing Center - Centro Nacional de Supercomputación. 
5.8

\section{List of Symbols}

$D(E, t) \quad$ Energy-dependent diffusion coefficient

$\hat{D}(t) \quad$ Diffusivity operator

$d_{\mathrm{t}} \quad$ Carbon nanotube diameter

$E_{\mathrm{F}} \quad$ Fermi energy, Fermi level

$E_{g} \quad$ Semiconductor band gap

$E_{q}(k) \quad$ Energy dispersion relation, band or subband

$f(E) \quad$ Fermi-Dirac distribution

$G_{0} \quad$ Conductance quantum

$\hat{G}(E) \quad$ Green's function operator

$G(E) \quad$ Energy-dependent conductance

$\hat{H} \quad$ Hamiltonian operator

I Moment of inertia/electric current (context dependent)

$k \quad$ Bending stiffness, spring constant/wavevector, wavenumber (context dependent)

L Carbon nanotube length

$\ell_{\mathrm{e}}(E) \quad$ Energy-dependent elastic mean free path

$\ell_{\varphi} \quad$ Phase coherence length

$(n, m) \quad$ Carbon nanotube chiral indices

$S \quad$ Thermopower

$T(E) \quad$ Transmission coefficient

$\hat{V} \quad$ Coupling Hamiltonian operator

$v_{\mathrm{F}} \quad$ Electronic Fermi velocity

$\hat{X}(t) \quad$ Position operator in Heisenberg representation

$Y \quad$ Young's modulus

$\hat{\Sigma}_{\lambda}(E) \quad$ Self-energy operator for a contact

$\varepsilon_{0} \quad$ Tight binding on-site energy parameter

$\hat{\Gamma}_{\lambda}(E) \quad$ Level broadening function, escape rate into a contact

$\gamma_{0} \quad$ Tight binding hopping integral parameter

$\rho(E) \quad$ Density of states

$\rho(\mathbf{r}) \quad$ Electron charge density

$\rho(\mathbf{r}, E) \quad$ Spectral measure operator in real-space representation

$\sigma, \pi \quad$ Atomic orbital hybrids or bonds

$\tau \quad$ Mean free time

$\tilde{\zeta} \quad$ Localization length

\section{References}

1 S. Iijima, Nature 354 (1991) 56.

2 R. Saito, G. Dresselhaus, M. Dresselhaus, Physical Properties of Carbon Nanotubes, Imperial College Press, London, 1998.
3 A. Loiseau, P. Launois, P. Petit, S. Roche, J.-P. Salvetat (eds.), Understanding Carbon Nanotubes From Basics to Applications, Lecture Notes in Physics, Springer Berlin, 677 (2006). 
4 P. J. F. Harris, Carbon Nanotubes and Related Structures, Cambridge University Press, Cambridge, 2002.

5 H. Terrones, M. Terrones, New J. Phys. 5 (2003) 126.1.

6 R. H. Baughman, A. A. Zakhidov,W. A. de Heer, Science, 297 (2002) 787.

7 J. Kong et al., Science, 287 (2000) 622.

8 P. G. Collins, K. Bradley, M. Ishigami, A. Zettl, Science, 287 (2000) 1801.

9 M. Burghard, Surf. Sci. Rep. 58 (2005) 1.

10 N. Sinha, J. Ma, J. T. W. Yeow, J. Nanosci. Nanotechnol. 6 (2006) 573.

11 E. S. Snow, F. K. Perkins, J. A. Robinson, Chem. Soc. Rev. 35 (2006) 790.

1212 P. Qi et al., Nano Lett. 3 (2003) 347.

13 C. Staii, A. T. Johnson, Nano Lett. 5 (2005) 1774

14 B. R. Goldsmith et al., Science, 315 (2007) 77.

15 E. Snow, F. Perkins, E. Houser, S. Badescu, T. Reinecke, Science, 307 (2005) 1942; E. Snow, F. Perkins, Nano Lett. 5 (2005) 2414.

16 S. Chopra, A. Pham, J. Gaillard, A. Parker, A. M. Rao, Appl. Phys. Lett. 80 (2002) 4632.

17 G. U. Sumanasekera et al., Phys. Rev. Lett. 89 (2004) 166801.

18 A. Cao et al., Adv. Mater. 17 (2005) 147.

19 D. A. Heller et al., Science, 311 (2006) 508.

20 P. Poncharal, Z. L. Wang, D. Ugarte, W. A. de Heer, Science, 83 (1999) 1513.

21 M. Nishio, S. Sawaya, S. Akita, Y. Nakayama, Appl. Phys. Lett. 86 (2005) 133111.

22 V. Sazonova, Y. Yaish, H. Üstünel, D. Roundy, T. A. Arias, P. L. McEuen, Nature, 431 (2004) 284.

23 B. Witkamp, M. Poot, H. S. J. van der Zant, Nano Lett. 6 (2006) 2904.

24 H. B. Peng, C. W. Chang, S. Aloni, T D. Yuzvinsky, A. Zettl, Phys. Rev. Lett. 97 (2006) 087203.

25 S. Ghosh, A. K. Sood, N. Kumar, Science, 299 (2003) 1042.

26 B. Bourlon, J. Wong, C. Mikó, L. Forró, M. Bockrath, Nat. Nanotechnol. 2 (2007) 104.

27 A. Modi, N. Koratkar, E. Lass, B. Wei, P. M. Ajayan, Nature, 424 (2003) 171.
28 J. Wang, Electroanalysis, 17 (2005) 7.

29 L. Roschier, R. Tarkiainen, M. Ahlskog, M. Paalanen, P. Hakonen, Appl. Phys. Lett. 78 (2001) 3295.

30 J.-P. Cleuziou, W. Wernsdorfer, V. Bouchiat, T. Ondarçuhu M. Monthioux, Nat. Nanotechnol. 1 (2006) 53.

31 M. S. Dresselhaus, G. Dresselhaus, P. C. Eklund, Science of Fullerenes and Carbon Nanotubes, Academic Press, San Diego, (1996) 965.

32 T. W. Ebbesen (ed.), Carbon Nanotubes: Preparation and Properties, CRC Press, Tokyo, (1997) 296.

33 P. Lambin, V. Meunier, A. Rubio, Phys. Rev. B 62 (2000) 5129.

$34 \mathrm{Ph}$. Lambin, V. Meunier, A. Rubio, in: Science and Application of Nanotubes, Fundamental Materials Research, M. F. Thorpe, D. Tománek, R. J. Enbody (eds.), Springer, Berlin, 2002.

35 J. A. Majewski, P. Vogl, Phys. Rev. B 35 (1987) 9666

36 W. Kohn, L. J. Sham, Phys. Rev. 140 (1965) A1133; P. Hohenberg, W. Kohn, Phys. Rev. B 136 (1964) B864.

37 S. Lunqvist, N. H. March (eds.), Theory of the Inhomogeneous Electron Gas, Plenum Press, New York, 1983; R. M. Dreizler, E. K. U. Gross, Density Functional Theory, an Approach to the Quantum Many Body Problem, Springer, Berlin, 1990; J. M. Seminario (ed.), Recent Developments and Applications of Modern Density Functional Theory, Elsevier, Amsterdam, 1996.

38 W. E. Pickett, Comput. Phys. Rep. 9 (1989) 115.

39 A. Szabo, N. S. Ostlund, Modern Quantum Chemistry. Introduction to Advanced Electronic Structure Theory, Dover, Mineola, NY, 1996.

40 L. Hedin, S. Lundqvist, in: Solid State Physics, H. Ehrenreich, F. Seitz, D. Turnbull (eds.), Academic Press, New York, 1969.

41 F. Aryasetiawan, O. Gunnarsson, Rep. Prog. Phys. 61 (1998) 237.

42 B. Farid, in: Electron Correlation in the Solid State, N. H. March (ed.), World Scientific, Imperial College Press, 1999. Nogueira, A. Rubio, K. Burke, E. K. U. Gross (Eds.), Time Dependent Density 
Functional Theory (TDDFT), Lecture Notes in Physics, Springer, Belin, 706, (2006).

44 G. Onida, L. Reinig, A. Rubio, Rev. Mod. Phys. 74 (2002) 601; F. Aryasetiawan, and O. Gunnarsson, Rep. Prog. Phys. 61 (1998) 237; W. G. Aulbur, L. Jönsson, J. W. Wilkins, Solid State Phys. 54 (1999) 1.

45 D. Prezzi, E. Molinari, Phys. Status Solidi A 203 (2006) 3602.

46 A. G. Marinopoulos, L. Reining, A. Rubio, and N. Vast, Phys. Rev. Lett. 91 (2003) 046402.

47 C. D. Spataru, S. Ismail-Beigi, L. X. Benedict, S. G. Louie, Phys. Rev. Lett. 92 (2004) 077402; E. Chang, G. Bussi, A. Ruini, E. Molinari, Phys. Rev. Lett. 92 (2004) 196401; E. Chang, G. Bussi, A. Ruini, E. Molinari, Phys. Rev. B 72 (2005) 195423.

48 M. P. Anantram, F. Léonard, Rep. Prog. Phys. 69 (2006) 507.

49 Y. Xue, S. Datta, M. A. Ratner, Chem. Phys. 281 (2002) 151; A. Nitzan, M. A. Ratner, Science 300 (2003) 1384; S. Kurth, G. Stefanucci, C. O. Almbladh, A. Rubio, E. K. U. Gross, Phys. Rev. B 72 (2005) 035308-1; B. G. Stefanucci et al., Time-Dependent Transport Through Single Molecules: Nonequilibrium Greens Functions and TDDFT, Lecture Notes in Physics, Springer, Berlin, 2006.

50 S. Datta, Electronic Transport in Mesoscopic Systems, Cambridge University Press, Cambridge, 1995.

51 G. Cuniberti, G. Fagas, K. Richter (eds.), Introducing Molecular Electronics, Lecture Notes in Physics, Springer, Berlin, 680 (2005); H. Haug, A.-P. Jauho, Quantum Kinetics in Transport and Optics of Semiconductors, Springer, Berlin, 1998.

52 K. S. Thygesen, A. Rubio, J. Chem. Phys. 126 (2007) 091101.

53 S. Roche and D. Mayou, Phys. Rev. Lett. 79 (1997) 2518; S. Roche, Phys. Rev. B 59 (1999) 2284; S. Roche, F. Triozon, A. Rubio and D. Mayou, Phys. Rev. B 64 (2001) 121401; S. Roche and R. Saito, Phys. Rev. Lett. 87 (2001) 246803; S. Roche, J. Jiang, F. Triozon, R. Saito, Phys. Rev. Lett. 95 (2005) 076803.
References 225

54 C. T. White, T. N. Todorov, Nature, 393 (1998) 240.

55 F. Triozon, S. Roche, A. Rubio, D. Mayou, Phys. Rev. B 69 (2004) 121410.

56 M. P. López Sancho, J. M. López Sancho, J. Rubio, J. Phys. F: Met. Phys. 15 (1985) 851.

57 F. Triozon, Ph. Lambin, S. Roche, Nanotechnology, 16 (2005) 230.

58 M. P. Anantram, T. R. Govindan, Phys. Rev. B 58 (1998) 4882.

59 F. Triozon, S. Roche, Eur. Phys. J. B 46 (2005) 427.

60 E. Akkermans, G. Montambaux, Physique Mésoscopique des Électrons et des Photons, EDP Sciences, 2004.

61 B. I. Yakobson, C. J. Brabec, J. Bernholc, Phys. Rev. Lett. 76 (1996) 2511.

62 J. Tersoff, Phys. Rev. B 37 (1998) 6991; J. Tersoff, R. S. Ruoff, Phys. Rev. Lett. 73 (1994) 676; D. W. Brenner, Phys. Rev. B 42 (1990) 9458.

63 M. J. Field, P. A. Bash, M. Karplus, J. Comp. Chem. 11 (1990) 700; B. R. Brooks et al., J. Comput. Chem. 2 (1983) 187.

64 C. M. Goringe, D. R. Bowler, E. Hernández, Rep. Prog. Phys. 60 (1997) 1447.

65 M. C. Payne, M. P. Teter, D. C. Allan, T. A. Arias, J. D. Joannopoulos, Rev. Mod. Phys. 64 (1992) 1045.

66 R. Carr, M. Parrinello, Phys. Rev. Lett. 55 (1985) 2471.

67 J. Tersoff, D. R. Hamann, Phys. Rev. Lett. 50 (1983) 1998.

68 A. Rubio, D. Sánchez-Portal, E. Artacho, P. Ordejón, J. M. Soler, Phys. Rev. Lett. 82 (1999) 3520.

69 A. Rubio, Appl. Phys. A 68 (1999) 275.

70 P. Delaney, H. J. Choi, J. Ihm, S. G. Louie, M. L. Cohen, Nature, 391 (1998) 466.

71 J. W. G. Wildöer, L. C. Venema, A. G. Rinzler, R. E. Smalley, C. Dekker, Nature, 391 (1998) 59; T. W. Odom, J.-L. Huang, P. Kim, C. M. Lieber, Nature, 391 (1998) 62.

72 M. Ouyang, J.-L. Huang, C. L. Cheung, C. M. Lieber, Science, 292 (2001) 702.

73 J. C. Charlier, X. Blase, S. Roche, Rev. Mod. Phys. in press.

74 A. Star et al., Nanolett. 3 (2003) 1421; A. Star et al., Macromolecules 36 (2003) 553; 
K. Balasubramanian et al., Nanolett. 4 (2004) 827.

75 T. Ando, T. Nakanishi, R. Saito, J. Phys. Soc. Jpn. 67 (1998) 2857-2862; P. L. McEuen et al., Phys. Rev. Lett. 83 (1999) 5098.

76 S. Roche, G. Dresselhaus, M. S. Dresselhaus, R. Saito, Phys. Rev. B 62 (2000) 16092.

77 Ch. Ewels, M. Glerup, J. Nanosci. Nanotechnol. 5 (2005) 1354.

78 H. J. Choi, J. Ihm, S. G. Louie, M. L. Cohen, Phys. Rev. Lett. 84 (2000) 2917.

79 C. C. Kaun et al., Phys. Rev. B 65 (2002) 205416; A. H. Nevidomskyy, G. Csanyi, M. C. Payne, Phys. Rev. Lett. 91 (2003) 105502; H.-F. Song, J.-L. Zhu, J.-J. Xiong, Phys. Rev. B 95 (2005) 216602.

80 S. Latil, S. Roche, D. Mayou, J. C. Charlier, Phys. Rev. Lett. 92 (2004) 256805.

81 Ch. Adessi, S. Roche, X. Blase, Phys. Rev. B 73 (2006) 125414.

82 R. Avriller, S. Latil, F. Triozon, X. Blase, S. Roche, Phys. Rev. B 74 (2006) 121406.

83 D. J. Thouless, Phys. Rev. Lett. 39 (1977) 1167; E. Abrahams et al., Phys. Rev. Lett. 42 (1979) 673.

84 V. H. Crespi, L. X. Benedict, M. L. Cohen, S. G. Louie, Phys. Rev. B 53 (1996) 13303; V. H. Crespi, M. L. Cohen, A. Rubio, Phys. Rev. Lett. 79 (1997) 2093.

85 C. Gómez-Navarro, P. J. De Pablo, J. Gómez-Herrero, B. Biel, F.-J. GarciaVidal, A. Rubio, F. Flores, Nat. Mater. 4 (2005) 534.

86 B. Biel, F. J. García-Vidal, A. Rubio, F. Flores, Phys. Rev. Lett. 95 (2005) 266801.

87 Y.-S. Lee, M. Buongiorno-Nardelli, N. Marzari, Phys. Rev. Lett. 95 (2005) 076804.

88 Y.-S. Lee, N. Marzari, Phys. Rev. Lett. 97 (2006) 116801.

89 D. Grimm, A. Latgé, R. B. Muniz, M. S. Ferreira, Phys Rev. B 71 (2005) 113408.

90 N. Ranjan, G. Seifert, Phys. Rev. B 73 (2006) 153408.

91 J. P. Small et al., Phys. Rev. Lett. 91 (2003) 256801.

92 F. Tournus, J. C. Charlier, Phys Rev. B 71 (2005) 165421. F. Tournus, S. Latil,
M. Heggie, J. C. Charlier, Phys Rev. B 72 (2005) 075431.

93 J. Zhao et al., Appl. Rev. Lett. 82 (2003) 3746; P. Gianozzi, Appl. Rev. Lett. 84 (2003) 3936; M. Arab et al., Phys. Rev. B 69 (2004) 165401.

94 S. Latil, S. Roche, J.-Ch. Charlier, NanoLett. 5 (2005) 2216.

95 P. R. Briddon, R. Jones, Phys. Status Solidi B 217 (2003) 131.

96 G. B. Bachelet, D. R Haman, M. Schlüter, Phys. Rev. B 26 (1982) 4199.

97 L. Salem, The Molecular-Orbital Theory of $\pi$-Conjugated Systems, Benjamin, Reading, MA, 1966.

98 A. V. Morozov, K. M. S. Misura, K. Tsemekhman, D. Baker, J. Phys. Chem. B 108 (2004) 8489.

99 F. L. Gervasio, P. Procacci, G. Cardini, A. Guarna, A. Giolitti, J. Phys. Chem. B 104 (2000) 1108

100 K. M. S. Misura, A. V. Morozov, D. Baker, J. Mol. Biol. 342 (2004) 651.

101 M. O. Sinnokrot, E. F. Valeev, C. D. Sherrill, J. Am. Chem. Soc. 124 (2002) 10887.

102 F. Ortmann, W. G. Schmidt, F. Bechstedt, Phys. Rev. Lett 95 (2005) 186101.

103 S. F. Boys, F. Bernardi, Mol. Phys. 19 (1970) 553.

104 L. Kale et al., J. Comp. Phys. 151 (1999) 283.

105 A. D. MacKerell Jr. et al., J. Phys. Chem. B 102 (1998) 3586.

106 J. M. Soler et al., J. Phys.: Condens Matter 14 (2002) 2745.

107 C. Roman, F. Ciontu, B. Courtois, Mol. Simul. 31 (2005) 123

108 C. Roman, F. Ciontu, B. Courtois, 4th IEEE Conference on Nanotechnolog, 263 (2004) 16-19.

109 S. Timoshenko, J. N. Goodier, Theory of Elasticity, McGraw-Hill, New York, 1983.

110 H. Dai, J. H. Hafner, A. G. Rinzler, D. T. Colbert, R. E. Smalley, Nature, 384 (1996) 147.

111 C. L. Cheung, J. H. Hafner, C. M. Lieber, Proc. Natl. Acad. Sci. USA 97 (2000) 3809.

112 P. Kim, C. M. Lieber, Science, 286 (1999) 2148.

113 M. Dequesnes, S. V. Rotkin, N. R. Aluru, Nanotechnology, 13 (2003) 120. 
114 D. Sanchez-Portal, E. Artacho, J. M. Soler, A. Rubio, P. Ordejon, Phys. Rev. B 59 (1999) 12678.

115 E. Hernandez, C. Goze, P. Bernier, A. Rubio, Appl. Phys. A 68 (1999) 287.
116 T. Belytschko, S. P. Xiao, G. C. Schatz, R. S. Ruoff, Phys. Rev. B 65 (2002) 235430.

117 W. H. Noon, K. D. Ausman, R. E. Smalley, J. P. Ma, Chem. Phys. Lett. 355 (2002) 445. 


\section{keywords/abstract}

Dear author,

Please check and/or supply keywords (3 to 5) and an abstract. These will not be included in the print version of your chapter but in the online version. If you do not supply an abstract, the section heading will be used instead.

Thank you!

Abstract

1 ..

Keywords

sensor-based devices; carbon nanotubes; modeling; transport phenomena 


\section{AUTHOR QUERIES}

Dear Author,

During the preparation of your manuscript for publication, the questions listed below have arisen. Please attend to these matters and return this form with your proof.

Many thanks for your assitstance.

\begin{tabular}{|c|c|c|}
\hline $\begin{array}{l}\text { Query } \\
\text { References }\end{array}$ & Query & Remarks \\
\hline 1 & $\begin{array}{l}\text { AU: Please supply an Abstract and check } \\
\text { Keywords. }\end{array}$ & \\
\hline 2 & AU: Please give Chapter number. & \\
\hline 3 & AU: Please give Chapter number. & \\
\hline 4 & AU: Please give Chapter number. & \\
\hline 5 & AU: Please give Chapter number. & \\
\hline 6 & AU: Please give Chapter number. & \\
\hline 7 & AU: Please give Chapter number. & \\
\hline 8 & AU: Please give Chapter number. & \\
\hline 9 & $\begin{array}{l}\text { AU: In this section, please check that the insets } \\
\text { are correctly referred to. }\end{array}$ & \\
\hline 10 & $\begin{array}{l}\text { AU: Figure 1a? Please give correct Figure } \\
\text { number. }\end{array}$ & \\
\hline 11 & $\begin{array}{l}\text { AU: There are no curves c and d in Figure 5.7. } \\
\text { Please correct. }\end{array}$ & \\
\hline 12 & $\begin{array}{l}\text { AU: Reference } 12 \text { and all other references with } \\
\text { "et al.": please give all names. }\end{array}$ & \\
\hline 13 & $\begin{array}{l}\text { AU: Reference 32: please give the authors of } \\
\text { the chapter on p. } 296 \text {. }\end{array}$ & \\
\hline 14 & AU: Reference 34: please give pages. & \\
\hline 15 & AU: Reference 40: please give pages. & \\
\hline
\end{tabular}




\begin{tabular}{|l|l|l|}
\hline 16 & $\begin{array}{l}\text { AU: Reference 42: you give two publishers. } \\
\text { Please give the correct publisher + location. } \\
\text { Also, please give pages. }\end{array}$ & \\
\hline 17 & $\begin{array}{l}\text { AU: Reference 60: please give location of EDP } \\
\text { Sciences. }\end{array}$ & \\
\hline 18 & AU: Reference 73: update? & \\
\hline
\end{tabular}

